\title{
When feedback fails: the scaling and saturation of star formation efficiency
}

\author{
Michael Y. Grudić, ${ }^{1 \star}$ Philip F. Hopkins, ${ }^{1}$ Claude-André Faucher-Giguère, ${ }^{2}$ \\ Eliot Quataert, ${ }^{3}$ Norman Murray ${ }^{4} \dagger$ and Dušan Kereš ${ }^{5}$ \\ ${ }^{1}$ TAPIR, Mailcode 350-17, California Institute of Technology, Pasadena, CA 91125, USA \\ ${ }^{2}$ Department of Physics and Astronomy and CIERA, Northwestern University, 2145 Sheridan Road, Evanston, IL 60208, USA \\ ${ }^{3}$ Department of Astronomy and Theoretical Astrophysics Center, University of California Berkeley, Berkeley, CA 94720, USA \\ ${ }^{4}$ Canadian Institute for Theoretical Astrophysics, 60 St George Street, University of Toronto, ON M5S 3H8, Canada \\ ${ }^{5}$ Department of Physics, Center for Astrophysics and Space Science, UC San Diego, 9500 Gilman Drive, La Jolla, CA 92093, USA
}

Accepted 2018 January 3. Received 2017 December 3; in original form 2016 December 16

\begin{abstract}
We present a suite of 3D multiphysics MHD simulations following star formation in isolated turbulent molecular gas discs ranging from 5 to 500 parsecs in radius. These simulations are designed to survey the range of surface densities between those typical of Milky Way giant molecular clouds (GMCs) $\left(\sim 10^{2} \mathrm{M}_{\odot} \mathrm{pc}^{-2}\right)$ and extreme ultraluminous infrared galaxy environments $\left(\sim 10^{4} \mathrm{M}_{\odot} \mathrm{pc}^{-2}\right)$ so as to map out the scaling of the cloud-scale star formation efficiency (SFE) between these two regimes. The simulations include prescriptions for supernova, stellar wind, and radiative feedback, which we find to be essential in determining both the instantaneous per-freefall $\left(\epsilon_{\mathrm{ff}}\right)$ and integrated $\left(\epsilon_{\mathrm{int}}\right)$ star formation efficiencies. In all simulations, the gas discs form stars until a critical stellar surface density has been reached and the remaining gas is blown out by stellar feedback. We find that surface density is a good predictor of $\epsilon_{\text {int }}$, as suggested by analytic force balance arguments from previous works. SFE eventually saturates to $\sim 1$ at high surface density. We also find a proportional relationship between $\epsilon_{\mathrm{ff}}$ and $\epsilon_{\text {int }}$, implying that star formation is feedback-moderated even over very short time-scales in isolated clouds. These results have implications for star formation in galactic discs, the nature and fate of nuclear starbursts, and the formation of bound star clusters. The scaling of $\epsilon_{\mathrm{ff}}$ with surface density is not consistent with the notion that $\epsilon_{\mathrm{ff}}$ is always $\sim 1$ per cent on the scale of GMCs, but our predictions recover the $\sim 1$ per cent value for GMC parameters similar to those found in spiral galaxies, including our own.
\end{abstract}

Key words: galaxies: active-galaxies: nuclei-galaxies: starburst-galaxies: star clusters: general-galaxies: star formation.

\section{INTRODUCTION}

Typically, star formation in the observed Universe is inefficient in any sense of the word. Star formation is observed to occur in giant molecular clouds (GMCs) formed in galactic discs, and the perfreefall star formation efficiency (SFE) of a star-forming region may be parametrized as

$\dot{M}_{\star}(t)=\epsilon_{\mathrm{ff}}(t) \frac{M_{\mathrm{gas}}(t)}{t_{\mathrm{ff}}(t)}$,

where $\dot{M}_{\star}$ is the star formation rate, $M_{\text {gas }}$ is the gas mass 'available' to form stars (observationally, the mass of molecular or dengas

${ }^{\star}$ E-mail: mgrudich@caltech.edu

†Canada Research Chair in Astrophysics. as obtained from a tracer such as $\mathrm{CO}$ or $\mathrm{HCN})$, and $t_{\mathrm{ff}}(t)$ is the local gravitational freefall time. $\epsilon_{\mathrm{ff}}$ is the fraction of available gas converted to stars per $t_{\mathrm{ff}}$; on galactic $(\sim \mathrm{kpc})$ scales, $\epsilon_{\mathrm{ff}}$ has been estimated by fitting to the relation:

$\Sigma_{\mathrm{SFR}}=\epsilon_{\mathrm{ff}}^{\mathrm{gal}} \Sigma_{\mathrm{gas}} t_{\mathrm{ff}}^{-1}$,

where $\Sigma_{\mathrm{SFR}}$ is the projected density of star formation in the disc, $\Sigma_{\text {gas }}$ is the projected (cold) gas density, $t_{\text {ff }}$ is the local freefall time evaluated from the galaxy's scale height-averaged density, and $\epsilon_{\mathrm{ff}}^{\mathrm{gal}}$ has been found to be $\sim 0.02$ (Kennicutt 1998b). Thus, a typical galaxy converts only 2 percent of its potentially starforming gas into stars each freefall time, despite the tendency of self-gravitating cold gas clouds to fragment and contract nearly all of their gas mass to high densities within only a few $t_{\mathrm{ff}}$. Clearly, some physical mechanism is responsible for the moderation of star formation. 
Recently, the FIRE ${ }^{1}$ (Feedback In Realistic Environments) simulations (Hopkins et al. 2014, 2017) have demonstrated that the inefficiency of star formation in galaxies formed within the $\Lambda \mathrm{CDM}$ cosmology can be explained by stellar feedback, obtaining good agreement with Kennicutt (1998b) independent of the numerical resolution-scale star formation model. As stars form in dense GMCs within a galaxy, some combination of photoionization heating, radiation pressure, stellar winds, and possibly supernovae blow out the remaining gas in the cloud, terminating star formation locally. The young stars formed inject momentum, mass, and energy into the surrounding ISM, which prevents the runaway vertical collapse of the galactic disc by providing turbulent support, and the rates of turbulent dissipation and momentum injection are in equilibrium when $\epsilon_{\mathrm{ff}}^{\mathrm{gal}} \sim 0.02$ (see Thompson, Quataert \& Murray 2005; Ostriker \& Shetty 2011; Faucher-Giguère, Quataert \& Hopkins 2013; Orr et al. 2017).

However, this mechanism only explains the rate of star formation on galactic scales: $\epsilon_{\mathrm{ff}}^{\mathrm{gal}}$ emerges from an established equilibrium the formation and disruption of many GMCs, and is distinct from the value of $\epsilon_{\mathrm{ff}}$ for a single GMC. Since star formation in a GMC must cease once it is disrupted, there exists another quantity of interest in characterizing the efficiency of star formation, the integrated SFE:

$\epsilon_{\text {int }}=\frac{M_{\star}}{M_{\mathrm{tot}}}$,

where $M_{\star}$ is the final mass of stars formed and $M_{\text {tot }}$ is the mass of the initial gas cloud. In Milky Way GMCs, the median value of $\epsilon_{\text {int }}$ is of the order of 1 per cent (Evans et al. 2009; Murray 2011; Lee, Miville-Deschênes \& Murray 2016; Vutisalchavakul, Evans \& Heyer 2016) with a large observed scatter of 0.8dex (Murray 2011; Lee et al. 2016). However, there is evidence that $\epsilon_{\text {int }}$ is much higher in denser conditions: Murray, Quataert \& Thompson (2010) points out that the masses of GMCs (Keto, Ho \& Lo 2005) and young star clusters (McCrady \& Graham 2007) in the M82 starburst galaxy are of a similar mass scale, suggesting that $\epsilon_{\text {int }}$ is of order unity at the greater surface densities of such regions. Indeed, the existence of young, bound star clusters in general may physically require high integrated SFE on at least some local scale (Tutukov 1978; Hills 1980; Elmegreen 1983; Mathieu 1983; Elmegreen \& Efremov 1997). Recent observations of young massive clusters (YMCs) have also suggested a time constraint of $<4$ Myr for cluster formation within the disc of M83 (Hollyhead et al. 2015), only twice the typical GMC freefall time in the central region of M83 (Freeman et al. 2017), suggesting that cluster formation may also be a dynamically fast process. Therefore, it is necessary to explore ways in which the efficiency of star formation, both in terms of $\epsilon_{\mathrm{ff}}$ and $\epsilon_{\text {int }}$, can scale from Milky Way like values of $\sim 1$ per cent to greater values. Since stellar feedback is responsible for the eventual disruption of molecular clouds against gravity, it is likely that the balance of these two forces plays a major role in determining both the speed and integrated efficiency of star formation at sub-kpc scales.

In this paper, we focus on the detailed behaviour of a single star formation episode at high resolution: we present 3D MHD simulations of star-forming gas discs which use the numerical treatments of cooling, star formation and stellar feedback of Hopkins et al. (2017) to answer certain basic questions about star formation in local galactic environments:

${ }^{1}$ http://fire.northwestern.edu (i) Given an initial self-gravitating gas distribution, what is the resulting star formation history? In particular, what determines the observable quantities $\epsilon_{\mathrm{ff}}$ and $\epsilon_{\text {int }}$, and how are they related?

(ii) How do the initial parameters of the gas cloud map on to the properties of the formed stellar system?

(iii) Which physical mechanisms have the greatest effect upon the answers to these questions?

The general approach of this study is to suppose some generic initial conditions for an isolated gas disc, neglecting its interaction with the surrounding galactic environment. This approximation makes sense for simulations spanning no more than a few dynamical times (which we shall show to be the case) and allows us to achieve relatively high spatial and mass resolution in the region of interest for modest computational cost.

This physics problem is most conventionally applicable to starforming GMCs, but really any region in which the dynamical time is not significantly longer than the main-sequence lifetime of massive stars ( $\sim 3 \mathrm{Myr}$ ) should be unstable to runaway star formation and the eventual blowout of the gas component (Torrey et al. 2017). The central regions of ultraluminous infrared galaxies (ULIRGs) may have large gas fractions and short dynamical times (Downes \& Solomon 1998; Bryant \& Scoville 1999), so for the purposes of our problem they may effectively behave as one super-GMC with particularly high $\left(>10^{3} \mathrm{M}_{\odot} \mathrm{pc}^{-2}\right)$ surface density. Our simulations, which probe these surface densities, can therefore also serve as models of gas-rich nuclear discs, which host the most extreme star formation events in the local Universe.

This paper is structured as follows: in Section 2, we describe a simple model of a gas-rich, star-forming disc, and predict its general behaviour from the physical arguments. In Section 3, we describe the methods for our simulations, their initial conditions, and the scope of our survey of physics and simulation parameters. In Section 4 we present the results of the simulations concerning the global properties of the star-forming clouds: the overall behaviour of the simulated clouds, the isolated effects of various physical mechanisms, the per-freefall $\left(\epsilon_{\mathrm{ff}}\right)$ and integrated $\left(\epsilon_{\mathrm{int}}\right)$ SFE. Finally, in Section 5 we discuss some applications, implications, and limitations of our results and outline future studies on the more detailed aspects of the mode of star formation we have simulated.

\section{A STAR-FORMING DISC MODEL}

To guide the methodology of the numerical study, we first review some basic theory of star formation and construct a simple model that captures the essential physics of how feedback determines the SFE of a gas-rich star-forming disc over short dynamical timescales. Consider an initially uniform disc of mass $M$, radius $R$, and scale height $h$ that initially consists of only gas. Averaged over the diameter of the disc, the initial surface density is then

$\Sigma_{\mathrm{tot}, 0}=\Sigma_{\mathrm{gas}}(t=0)=\frac{M}{\pi R^{2}}$.

\subsection{Time-scales for star formation}

The longest possible time-scale for gravitational collapse within the model disc is the freefall time $t_{\mathrm{ff}, 0}$ derived from the system's physical parameters $M$ and $R$ :

$t_{\mathrm{ff}, 0}=\frac{\pi}{2} \sqrt{\frac{R^{3}}{2 G M}}=2 \operatorname{Myr}\left(\frac{R}{50 \mathrm{pc}}\right)^{\frac{1}{2}}\left(\frac{\Sigma_{\mathrm{tot}, 0}}{10^{3} \mathrm{M}_{\odot} \mathrm{pc}^{-2}}\right)^{-\frac{1}{2}}$, 
which is proportional to the outer orbital period of the disc. This is the longest relevant time-scale in the problem, since we neglect environmental interactions. $t_{\mathrm{ff}, 0}$ may overestimate the typical gravitational collapse time of a typical gas parcel, as we expect that if star formation is to occur then the dynamics are driving mass to greaterthan-average densities with correspondingly shorter freefall times. Specifically, isothermal, self-gravitating turbulence has been found to produce a density PDF with a high-density power-law tail due to gravity (Kritsuk, Norman \& Wagner 2011), and at lower densities a lognormal form, as emerges in isothermal turbulence without gravity (Vazquez-Semadeni 1994; Padoan, Nordlund \& Jones 1997; Nordlund \& Padoan 1999). The only characteristic density is the peak of this distribution, so we define a shorter freefall time in terms of the median gas density $\rho_{50}$ (equivalently, number density $n_{50}$ ): ${ }^{2}$

$t_{\mathrm{ff}, 50}=\sqrt{\frac{3 \pi}{32 G \rho_{50}}}=1.6 \mathrm{Myr}\left(\frac{n_{50}}{10^{3} \mathrm{~cm}^{-3}}\right)^{-\frac{1}{2}}$,

where $n_{50}$ is the median particle number density. $t_{\mathrm{ff}, 50}$ will generally be a more reasonable unit for the gas depletion time, and hence for comparing values of $\epsilon_{\mathrm{ff}}$.

In the parameter space relevant to star formation in the local Universe, the cooling time of gas that is metal-enriched or molecular is generally much less than both $t_{\mathrm{ff}, 0}$ and $t_{\mathrm{ff}, 50}$. Therefore, in absence of stars or external inputs, any thermal energy supporting against self-gravity will quickly radiate away. If the disc has some initial turbulent velocity dispersion, that energy too will be cooled away by shocks over $\sim t_{\mathrm{ff}, 50}$. Without some imposed stabilizing force the disc will be subject to gravitational instability, fragmentation, and star formation.

The process of fragmentation involves a runaway collapse to protostellar densities. If an initially smooth disc with $\rho \sim \Sigma_{\text {tot, } 0} / 2 h$ were to fragment hierarchically into successively denser structures, the entire conversion of gas into stars would take no longer than a time of the order of $\sim t_{\mathrm{ff}, 50}$, since the freefall time at all smaller scales is less than this. Counting the time for the initial growth of the gravitational instability, and the eventual gas evacuation due to feedback, we expect the entire period of star formation to last no longer than several freefall times (e.g. Elmegreen 2000, 2007). This appears to be the case for Milky Way GMCs, which have a mean star-forming lifetime of 3 freefall times (Murray 2011; Lee et al. 2016), as well as those found in simulated galaxies with low-temperature cooling and stellar feedback (Hopkins, Quataert \& Murray 2012a), however it has also been argued that star formation should take longer (Tan et al. 2006; Krumholz \& Tan 2007).

\subsection{Star formation efficiency}

As stars form, the stellar surface density $\Sigma_{\star}(t)$ increases as the gas surface density $\Sigma_{\text {gas }}(t)=\Sigma_{\text {tot, } 0}-\Sigma_{\star}(t)$ decreases. These stars will inject energy and momentum into the gas through various feedback mechanisms, however if the time-scale of star formation is so short that $\mathrm{SNe}$ do not occur then direct ISM heating can be neglected due

\footnotetext{
${ }^{2}$ Note that we use the median, and not the mass-weighted mean gas density used for determining $t_{\mathrm{ff}}$ in Krumholz, Klein \& McKee (2011) and Myers et al. (2014). The mass-weighted mean is less suitable for estimating $t_{\mathrm{ff}}$ in the middle of star formation because the high-density power-law tail in the density PDF biases it towards high densities. We also find that it is not robust with respect to simulation resolution, as higher resolutions will resolve more of the power-law tail. The median density generally lies near the peak of the density PDF, and is robust with respect to resolution.
}

to the short cooling time. Assuming that the stellar population is well-sampled from a Kroupa (2002) IMF, the rate of momentum feedback injection per unit stellar mass $\frac{\dot{P}_{\star}}{m_{\star}}$ will initially be roughly constant, dominated by radiation pressure and fast winds from the most massive stars for the first $3 \mathrm{Myr}$ after the stellar population forms. For the subsequent $\sim 40 \mathrm{Myr}$, the massive stars all leave the main sequence and supernovae become the dominant form of feedback. Because we are most interested in the limit of dense systems with short dynamical times, we can neglect stellar evolution and approximate $\frac{\dot{P}_{\star}}{m_{\star}}$ as being constant. Then the force of feedback upon the gas in the disc is

$F_{\mathrm{fb}}(t)=\frac{\dot{P}_{\star}}{m_{\star}} M_{\star}=\frac{\dot{P}_{\star}}{m_{\star}} \Sigma_{\star}(t) \pi R^{2}$,

assuming no leakage, photon trapping, or other effects arising from clumpy structure. This force will continue to increase until $F_{\mathrm{fb}}$ exceeds the force of gravity binding the gas to the disc. The majority of the new star formation will occur in a thin disc, so while the gas is dense enough to form stars the gravitational field binding gas to the star-forming region will be dominated by contributions from the gas itself and the newly formed stars. Thus,

$F_{\mathrm{g}}(t)=g M_{\mathrm{gas}}(t)=2 \pi G \Sigma_{\mathrm{tot}, 0} \Sigma_{\mathrm{gas}}(t) \pi R^{2}$.

By equating the force of feedback upon the gas (7) with that of gravity (8) we can determine the final stellar mass and hence the integrated SFE (Fall, Krumholz \& Matzner 2010):

$\epsilon_{\text {int }}=\frac{M_{\star}}{M}=\frac{\Sigma_{\text {tot }, 0}}{\Sigma_{\text {tot }, 0}+\Sigma_{\text {crit }}}$,

where

$\Sigma_{\text {crit }}=\frac{1}{2 \pi G} \frac{\dot{P}_{\star}}{m_{\star}}$

is the quantity with units of surface density encoding the strength of feedback relative to gravity. The contributions to $\frac{\dot{P}_{\star}}{m_{\star}}$ from radiation pressure, stellar winds, and $\mathrm{SNe}$ ejecta (ignoring the work done in the energy-conserving phase) are all of order $10^{3} \frac{\mathrm{L} \odot}{\mathrm{M}_{\odot}{ }^{c}}$. Thus, $\Sigma_{\text {crit }} \sim 10^{3-4} \mathrm{M}_{\odot} \mathrm{pc}^{-2}$ due to stellar feedback physics. Observationally, the average $\epsilon_{\text {int }}$ for Milky Way GMCs is $\sim 3$ per cent (Murray 2011; Lee et al. 2016), while the median GMC surface density is $\sim 100 \mathrm{M}_{\odot} \mathrm{pc}^{-2}$ (Larson 1981; Solomon et al. 1987; Bolatto et al. 2008), so we can estimate that $\Sigma_{\text {crit }}=3000 \mathrm{M}_{\odot} \mathrm{pc}^{-2}$ for those GMCs for which feedback from massive stars is important. See also Murray et al. (2010), Dekel \& Krumholz (2013), and Thompson \& Krumholz (2016) for similar derivations with various cloud and feedback models.

Equation (9) predicts that the efficiency of starbursts occurring over adequately short time-scales is simply dictated by the ratio of forces of feedback and gravitation. In the limit $\Sigma_{\text {tot, } 0} \ll \Sigma_{\text {crit }}$, SFE is proportional to $\epsilon_{\text {int }} \propto \Sigma_{\text {tot, } 0}$ with the constant of proportionality determined by the strength of feedback. Inversely, where $\Sigma_{\text {tot, } 0} \gg \Sigma_{\text {crit }}$, SFE should approach unity: gravity prevails against feedback and converts nearly all gas to stars. The importance of surface density in determining SFE in short dynamical time systems is not simply a consequence of the 'diskiness' of star-forming systems, nor of their optical depth in some band, both of which would give surface density an obvious physical relevance. It is merely a consequence of the fact that the ratio between the force of selfgravity $F_{\mathrm{g}} \sim \frac{G M^{2}}{R^{2}}$ and the momentum injection rate of feedback $F_{\mathrm{fb}} \sim M_{\star} \dot{P}_{\star} / m_{\star}$ has dimensions of surface density, at least under our simplifying assumptions. 


\section{SIMULATIONS}

Our simulations use GIZMO (Hopkins 2015), ${ }^{3}$ a mesh-free, Lagrangian finite-volume Godunov code designed to capture advantages of both grid-based and smoothed-particle hydrodynamics (SPH) methods, built on the gravity solver and domain decomposition algorithms of GADGET-3 (Springel 2005). In Hopkins (2015) and Hopkins \& Raives (2016) we consider extensive surveys of test problems in both hydrodynamics and MHD, and demonstrate accuracy and convergence in good agreement with well-studied regular-mesh finite-volume Godunov methods and moving-mesh codes (e.g. ATHENA and AREPO; Stone et al. 2008; Springel 2010). We run GIZMO in its meshless-finite mass mode but have verified that meshless-finite volume mode produces nearly identical results (as expected from the previous studies).

\subsection{Cooling, star formation, and stellar feedback}

The simulations here use the physical models for star formation and stellar feedback developed for the FIRE project (Hopkins et al. 2014, 2017), although the simulations in this paper are idealized cloud collapse experiments on small scales, at often much higher mass resolution than the FIRE simulations. In general, we expect these methods to be appropriate to the scales examined in this work because by construction the FIRE framework adopts a physics approach that requires no phenomenological tuning to different mass scales. Hydrodynamics, gravity, cooling, and stellar feedback are explicitly and approximately solved down to the resolution limit, and the physics approximations invoked have been extensively validated by more expensive and detailed simulations. We briefly summarize some key properties of the FIRE models here, but refer to Hopkins et al. (2017) for details of the numerical implementations and extensive tests of the algorithms and physics.

When simulating gas fragmentation, it is critical to have explicit cooling physics; we therefore do not adopt an 'effective equation of state' (Springel \& Hernquist 2003) as has been done in many works in the past, but explicitly follow a wide range of heating/cooling processes. This includes photoionization and photoelectric, dust collisional, Compton, metal-line, molecular, and fine-structure processes, and we self-consistently account for optically thick cooling when local regions become thick to their own cooling radiation, implementing the approximation of Rafikov (2007). We do neglect the effects of non-equilibrium chemistry in the ISM, which can be very important for predictions of observational tracer abundances (Richings, Schaye \& Oppenheimer 2014a,b), however cooling times are generally so short in our problem that little dynamical effect can be expected.

Gas particles are converted to star particles with constant probability per unit time $t_{\mathrm{ff}}(\rho)^{-1}$ if they satisfy all of the following star formation criteria:

(i) Self-shielding and molecular: We compute the molecular fraction $f_{\text {mol }}$ of the gas as a function of column density and metallicity according to Krumholz \& Gnedin (2011), estimating the local gas column density with a Sobolev-like estimator.

(ii) Contracting: Star formation occurs only in regions of increasing density $(\nabla \cdot v<0)$.

(iii) Self-gravitating: The local Jeans mass $M_{\text {jeans }}$ is estimated, accounting for both turbulent (Hopkins, Narayanan \& Murray 2013)

\footnotetext{
${ }^{3} \mathrm{~A}$ public version of this code is available at www.tapir.caltech.edu /phopkins/Site/GIZMO.html
}

and thermal contributions, with the turbulent contribution typically dominating in cold molecular gas. Star formation is allowed only in regions where the Jeans mass can no longer be resolved, as it is at this point that fragmentation should continue down to unresolved scales.

In our tests, we find that the self-gravity criterion is the most restrictive and the most physically motivated of the above. Note that these criteria are slightly different from the FIRE simulations (Hopkins et al. 2014, 2017), as we do not enforce a threshold density for star formation, and require gas to be increasing in density to form stars. All star formation criteria are fully adaptive, with no built-in scales that could be imprinted upon the star clusters that form. To summarize, gas fragmentation is explicitly followed down to the scale where the mass resolution is insufficient to resolve fragmentation, then the gas particles quickly (within one local $t_{\mathrm{ff}}$ ) transition into collisionless star particles.

Crucially, because the collapse time-scale of resolved fragments at densities much larger than the mean in our simulations is always fast compared to the global dynamical time, this is not the ratelimiting step for star formation. Rather, it is the initial formation of these fragments (Thompson et al. 2005; Ostriker \& Shetty 2011; Faucher-Giguère et al. 2013). As such, we will show that the star formation histories are insensitive to details of both our cooling and star formation prescriptions. This is consistent with a wide range of previous studies on GMC and galactic scales (Saitoh et al. 2008; Hopkins, Quataert \& Murray 2011, 2012a,b; Agertz et al. 2013; Hopkins et al. 2016, 2017).

Once stars form, feedback is included in the form of radiation pressure (UV, optical, and IR), stellar winds (fast, young star winds and slow AGB winds), SNe (Types Ia and II), photoionization, and photoelectric heating. Every star particle is treated as a single stellar population with an age based on its formation time and metallicity and mass inherited from its parent gas particle. Feedback includes the relevant mass, metal (with 11 separately tracked species), momentum, and energy injection to the neighbouring gas; all of the relevant quantities (stellar luminosities, spectral shapes, SNe rates, wind mechanical luminosities, yields) for the mechanisms above are tabulated as a function of time directly from the stellar population models in STARBURST99, assuming a Kroupa (2002) IMF. For $\mathrm{SNe}$, if we lack the mass resolution to resolve the Sedov-Taylor phase, we estimate the work done during the energy-conserving phase and couple the appropriate momentum based on fits from high-resolution SNR simulations (Kim \& Ostriker 2015; Martizzi, Faucher-Giguère \& Quataert 2015, see Hopkins et al. 2014 for implementation details). This is only important for our few simulations with resolved masses greater than $10^{3} \mathrm{M}_{\odot}$.

For the multiband radiative fluxes necessary for the radiative heating and pressure terms, we use the LEBRON approximation, described in detail in Hopkins et al. (2017). The spectrum is binned into UV, optical/near-IR, and mid/far-IR bands, and the approximate fluxes are computed explicitly at each particle. Local extinction around star particles is estimated with an effective column density computed with a Sobolev approximation; the robustness of our results to unknown order-unity factors in this prescription is demonstrated in Appendix A2. We emphasize that, unlike the model of Hopkins et al. (2012a), LEBRON does not invoke a subgrid 'boost' term for the radiation pressure of multiply-scattered IR photons. Only explicitly resolved photon absorption is accounted for in the heating and pressure terms.

We intentionally assign IMF-averaged properties to all star particles, rather than attempting to follow individual stars 
Table 1. Initial conditions, numerical parameters, and modifications of the simulations in this paper: (1) $\Sigma_{\text {tot, } 0}$ : the initial average gas surface density in $\mathrm{M}_{\odot} \mathrm{pc}^{-2}$. (2) $R$ : the radius of the initial spherical gas cloud in pc. (3) $M$ : the initial gas mass in $\mathrm{M}_{\odot}$. (4) The freefall time $t_{\mathrm{ff}, 0}$ at the initial density, defined in equation (5). (5) Modifications to the simulation with respect to the standard setup described in Section 3. (6) Particle mass resolution in $\mathrm{M}_{\odot}$. (7) Minimum Plummer-equivalent force softening for star particles. No minimum softening for gas particles is imposed. The particle number is $100^{3}$ in all simulations unless otherwise specified. All simulations start with solar metal abundances (except where stated otherwise), and an initial temperature of $10^{4} \mathrm{~K}$.

\begin{tabular}{|c|c|c|c|c|c|c|}
\hline $\begin{array}{l}\Sigma_{\mathrm{tot}, 0}\left(\mathrm{M}_{\odot} \odot \mathrm{pc}^{-2}\right) \\
\text { (1) }\end{array}$ & $\begin{array}{l}R(\mathrm{pc}) \\
(2)\end{array}$ & $\begin{array}{l}M\left(\mathrm{M}_{\odot}\right) \\
(3)\end{array}$ & $\begin{array}{l}t_{\mathrm{ff}, 0}(\mathrm{Myr}) \\
(4)\end{array}$ & $\begin{array}{l}\text { Simulation parameters } \\
\text { Modifications } \\
\text { (5) }\end{array}$ & $\begin{array}{l}\text { Mass resolution }\left(\mathrm{M}_{\odot}\right) \\
\text { (6) }\end{array}$ & $\begin{array}{l}\text { Minimum star particle softening (pc) } \\
\text { (7) }\end{array}$ \\
\hline 127 & 5 & $10^{4}$ & 1.85 & & 0.03 & 0.001 \\
\hline 127 & 500 & $10^{8}$ & 18.53 & & 300 & 0.1 \\
\hline 382 & 5 & $3 \times 10^{4}$ & 1.07 & & 0.03 & 0.001 \\
\hline 1270 & 5 & $10^{5}$ & 0.59 & & 0.1 & 0.001 \\
\hline 1270 & 50 & $10^{7}$ & 1.85 & 'Standard' & 10 & 0.01 \\
\hline 1270 & 50 & $10^{7}$ & 1.85 & Random IC seeding 2 & 10 & 0.01 \\
\hline 1270 & 50 & $10^{7}$ & 1.85 & Random IC seeding 3 & 10 & 0.01 \\
\hline 1270 & 50 & $10^{7}$ & 1.85 & Optically thin cooling & 10 & 0.01 \\
\hline 1270 & 50 & $10^{7}$ & 1.85 & $50^{3}$ particle resolution & 80 & 0.01 \\
\hline 1270 & 50 & $10^{7}$ & 1.85 & 1 per cent local SFR & 10 & 0.01 \\
\hline 1270 & 50 & $10^{7}$ & 1.85 & $0.01 \mathrm{Z}_{\odot}$ initial metallicity & 10 & 0.01 \\
\hline 1270 & 500 & $10^{9}$ & 5.86 & & 1000 & 0.1 \\
\hline 3820 & 5 & $3 \times 10^{5}$ & 0.34 & & 0.3 & 0.001 \\
\hline 3820 & 50 & $3 \times 10^{7}$ & 1.07 & & 30 & 0.01 \\
\hline 3820 & 500 & $3 \times 10^{9}$ & 3.38 & & 3000 & 0.1 \\
\hline 12700 & 5 & $10^{6}$ & 0.19 & & 1 & 0.001 \\
\hline 12700 & 50 & $10^{8}$ & 0.59 & & 100 & 0.01 \\
\hline 12700 & 500 & $10^{10}$ & 1.85 & & 10000 & 0.1 \\
\hline
\end{tabular}

explicitly - our goal is to study the effects of feedback, given some IMF, not to solve the problem of the origins and nature of the IMF itself. The latter would require a full model for individual star formation (and much higher resolution than we are able to achieve here), and may critically depend on additional physics (e.g. heating by prostellar accretion, protostellar jets) which are negligible in an IMF-averaged feedback scenario. ${ }^{4}$ In some of our less-massive simulated clouds, the particle mass is less than $\mathrm{M}_{\odot}$ and the stellar IMF is nominally resolvable, so star formation tends to produce 'clusters' of star particles of $100 \mathrm{M}_{\odot}$ or less, which can be identified with the individual stars that would have formed. In this case, a

\footnotetext{
${ }^{4}$ One might worry that, by IMF-averaging, we make feedback 'too smooth.' In limited experiments, we have crudely modelled the effects of stochastic sampling of the IMF and concentrating feedback in individual massive stars by, for each star particle, drawing from the IMF a quantized number of massive O-stars (from a Poisson distribution with mean equal to the expectation for the total mass of the particle). All feedback effects associated with massive stars (Type-II SNe, photoheating, fast winds, radiation pressure) are multiplied appropriately by the number of O-stars (which are lost in each Type-II SNe event). As expected, this has essentially no effect on the disc-averaged properties we consider here for disc masses $\gtrsim 1000 \mathrm{M}_{\odot}$, which reasonably sample massive $\left(\gtrsim 10 \mathrm{M}_{\odot}\right)$ stars. For still smaller clouds, this (as expected) introduces additional scatter in the SFE, corresponding to the variation in the number of massive stars (hence strength of feedback). However, the mean scalings are unaffected.
}

sink-particle method (e.g. Bate, Bonnell \& Price 1995) is certainly much more realistic and efficient, however we still adopt the standard star-particle method for consistency with the more massive clouds.

\subsection{Initial conditions and problem setup}

The initial conditions of the simulations consist of a constant density gas sphere of radius $R$ and mass $M$, with the parameter space of $R$ and $M$ tabulated in Table 1 . These values are chosen to cover a range of values of $\Sigma_{\text {tot, } 0}$, which, for reasons discussed in Section 2, we expect to roughly parametrize the overall behaviour of the system even at disparate spatial scales, masses, and dynamical times.

The initial velocity field is a superposition of solid-body rotation about the origin and a random turbulent component. The rotational frequency is set to the gas ball's Keplerian frequency $\Omega_{\mathrm{K}}=\left(G M / R^{3}\right)^{\frac{1}{2}}$, so that the effective radius, and hence average surface density of the disc remains roughly constant. ${ }^{5}$ The random velocity component adds a turbulent energy of 10 percent of

\footnotetext{
${ }^{5}$ Note that assuming rotational support is not a realistic choice for simulating GMCs, which are generally supported by a shearing velocity gradient and turbulence. As such, the simulations are not expected to result in largescale cloud morphologies resembling realistic galactic GMCs. However, the morphology of sub-clouds will be determined on much shorter time-
} 


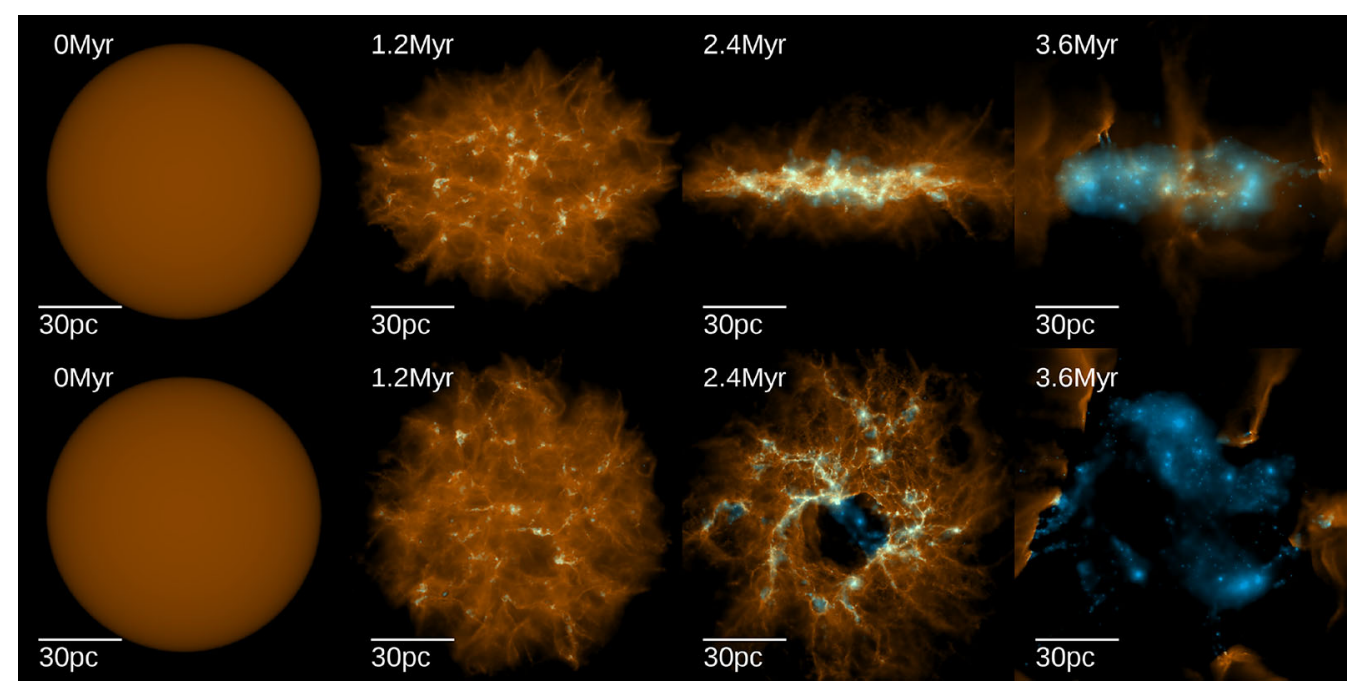

Figure 1. Surface density of gas (orange) and stars (blue) in our fiducial run with parameters $M=3 \times 10^{7} \mathrm{M}_{\odot}$ and $R=50$ pc, projected parallel (top row) and normal (bottom row) to the disc plane. Far left: The initial conditions, a uniform-density sphere. Centre left: After a time $\sim t_{\mathrm{ff},} 0=1.2 \mathrm{Myr}$, star formation has begun. Centre right: After another $t_{\mathrm{ff}, 0}$ has passed, the star formation rate has peaked and large star clusters have appeared. Far right: The system has reached the critical stellar mass, at which point the gas is blown out of the system by feedback, evacuating the central region.

the initial gravitational binding energy, with a power spectrum $E(k) \propto k^{-2}$. All velocity Fourier coefficients for which $\|\boldsymbol{k}\| \geq \frac{2 \pi}{R}$ are given a random phase and scaled according to this relation. The velocity components are first computed on a Cartesian grid circumscribing the gas sphere, and are then interpolated to the particle positions.

The seed magnetic field is constructed in a similar fashion, such that the power spectrum of magnetic energy is also proportional to $k^{-2}$. The only difference from the above is that the $\nabla \cdot \boldsymbol{B}$ constraint is enforced by first computing random Fourier coefficients for the magnetic potential $\boldsymbol{A}$ and then applying the curl operator in Fourier space before transforming to real space in the same fashion as the velocity. The total magnetic energy is 1 per cent of the gravitational binding energy, which is 10 per cent of the initial turbulent energy. This figure was chosen based upon observations suggesting that MHD turbulence in GMCs is super-Alfvénic (Troland \& Crutcher 2008), supported by high-resolution MHD simulations showing that the supersonic turbulent MHD dynamo tends to saturate the magnetic energy to $1-10$ percent of the turbulent energy (Federrath et al. 2014).

The gas is initialized to a temperature of $10^{4} \mathrm{~K}$, however the simulations' results are insensitive to this choice because the cooling time in all cases considered is orders of magnitude shorter than the dynamical time-scale. At the beginning of the simulation, the gas immediately cools rapidly to several tens of $\mathrm{K}$, as is typical of the cold, neutral phase of the interstellar medium.

All simulations except those noted in Table 1 have $10^{6}$ particles, giving a fixed mass resolution of $10^{-6} \mathrm{M}$. As discussed in Appendix A1, the star formation histories of the simulations are insensitive to our mass resolution at or above this level.

\section{RESULTS}

Qualitatively, all simulations follow the sequence of events illustrated in Fig. 1. The turbulent gas cloud immediately cools, with the

scales by local turbulence and self-gravity, independently of the large-scale morphology.

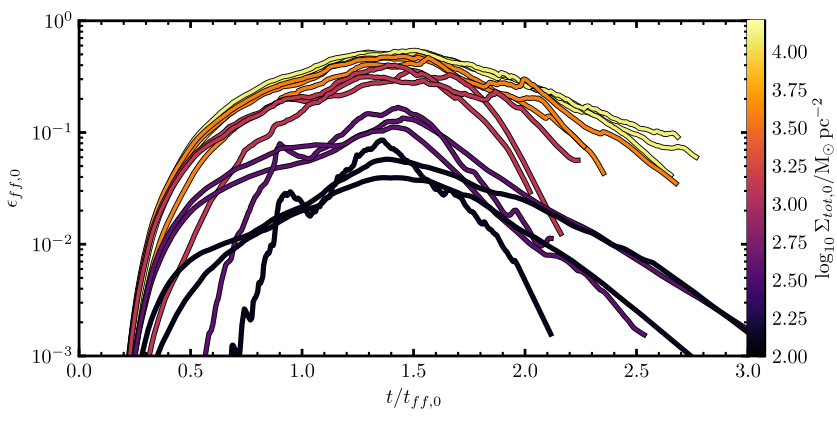

Figure 2. Dimensionless star formation histories of all parameter survey runs: the per-freefall SFE $\epsilon_{\mathrm{ff}, 0}=\frac{\dot{M}_{\star} t_{\mathrm{ff}, 0}}{M_{\mathrm{gas}}}$ as a function of time in units of the initial freefall time $t_{\mathrm{ff}, 0}$ for the respective run. Each curve is a single run, coloured according to the value of $\Sigma_{\text {tot, } 0}$. In all cases, $\epsilon_{\mathrm{ff}, 0}$ rises to a maximum dictated by the strength of feedback relative to self-gravity, saturating to a value of the order of 1 as $\Sigma_{\text {tot, } 0}$ gets large.

lowest temperatures reaching $\sim 10 \mathrm{~K}$. The initial velocity and magnetic fields seed density fluctuations and the gravitational instability grows, condensing the cloud into filaments and clumps. Within a freefall time, the first star clusters have formed. The star formation rate accelerates over $\sim t_{\mathrm{ff}, 0}$ to a peak value $\mathrm{SFR}_{\max } \propto \epsilon_{\mathrm{ff}} M / t_{\mathrm{ff}}$, with most star formation occurring in dense molecular sub-clouds (See Fig. 2). At this point the moderating effect of feedback comes into play and the SFR starts to drop as the disc acquires significant turbulent support. Eventually, all gas is blown out of the central region by feedback and star formation ceases. The product of the starburst is invariably a population of star clusters, some of which disperse upon gas expulsion, and some of which persist to the end of the simulation and remain bound. The end result is a population of star clusters surrounded by a diffuse, expanding gas shell.

\subsection{Effects of different physics}

In Fig. 3, we compare the star formation histories of the simulations evolved from identical initial conditions but with different physics enabled or disabled. It can be readily seen that the effect of varying 


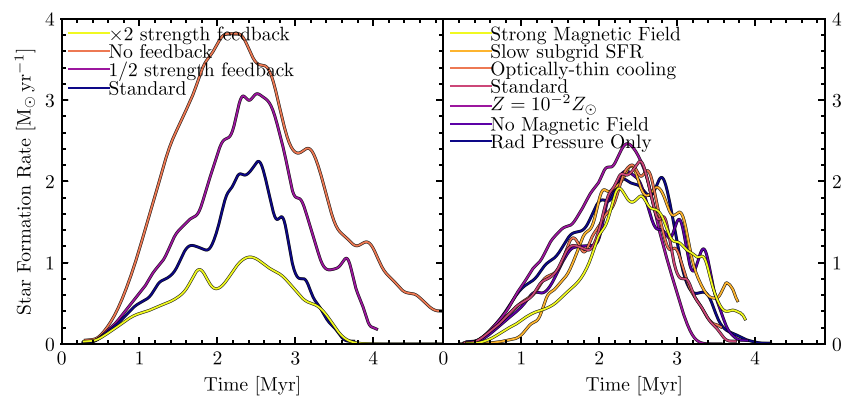

Figure 3. Star formation histories of the physics test runs using the standard initial parameters $M=10^{7} \mathrm{M}_{\odot}$ and $R=50$ pc. Left: Runs re-scaling the energy and momentum loadings of all stellar feedback mechanisms, producing large variations in the star formation history. Right: Our 'standard' run compared to runs evolved from the same initial conditions with various physics options: (1) Strong magnetic field: Setting the initial magnetic energy to 10 per cent of the binding energy, 10 times greater than standard. (2) Slow sub-grid SFR: artificially 'slowing' star formation in gas that satisfies the star formation criteria (Section 3.1) by multiplying the SFR by $1 / 100$. (3) Optically thin cooling: treating all radiative cooling as optically thin. (4) $Z=10^{-2} Z_{\odot}$ : lowering the initial metallicity from $\mathrm{Z}_{\odot}$ to $0.01 \mathrm{Z}_{\odot}$. (5) No magnetic field: turning off magnetic fields. (6) Rad pressure only: Removing all stellar feedback physics other than radiation pressure. These all produce relatively weak effects compared to simply rescaling the feedback energy and momentum fluxes, as discussed in Section 4.1.

the strength of feedback dwarfs all others, analogous to the conclusions of Su et al. (2017) for galaxy-scale star formation. Here, we enumerate and describe these modifications and explain why, physically, this should be the case.

\subsubsection{Stellar feedback}

In one run, we neglect feedback altogether, and in two others we scale all energy and momentum feedback rates by $1 / 2$ and 2 , respectively. We find that without any feedback moderation, star formation consumes nearly all ( 86 per cent by the end of the simulation) gas within $\sim 2 t_{\mathrm{ff}, 0}$, with no sign of stopping. If the strength of feedback is scaled by $1 / 2$, the SFE nearly doubles, while it is roughly halved when feedback is twice as strong, in agreement with equation (9). The time-scale for star formation remains unchanged, so the average per-freefall SFE $\epsilon_{\mathrm{ff}}$ is also determined by the strength of feedback

We also perform a run in which radiation pressure is the only feedback mechanism, and find that there is only marginally $(<10$ per cent $)$ more star formation than the standard run. Thus, radiation pressure accounts for most of the feedback budget at this point in parameter space. We expect this to be generally true in clouds where the dynamical time does not greatly exceed 3 Myr. Photoionization heating may have a significant contribution to disrupting the cloud if its escape velocity is $<10 \mathrm{~km} \mathrm{~s}^{-1}$ (Dale, Ercolano \& Bonnell 2012), but this will be the case for only a couple points in the parameter space of this paper.

It is clear from the first panel of Fig. 3 that the strength of feedback does not merely set the termination time of star formation: it also limits the star formation rate in an instantaneous sense - the stronger the feedback, the lesser the peak star formation rate. The specific feedback mechanism responsible for this is radiation pressure from young massive stars, as demonstrated by the radiationpressure-only run. The radiation pressure is able to halt accretion on to cluster-forming cores, terminating star formation locally while it is still ongoing globally. Supernova feedback does not have this instantaneous effect due to its inherent time lag after initial star formation. Although we have not simulated it, a hypothetical starburst with only supernova feedback would proceed much like the zero-feedback run for the first $3 \mathrm{Myr}$, which in this case is enough time to convert nearly all gas into stars. We therefore conclude that the early feedback mechanisms from massive stars are crucial in setting the efficiency of rapid star formation in the high-density, short dynamical time regime studied in this work.

\subsubsection{Optically thin cooling}

In one test run, we treating all radiative cooling as optically thin [i.e. ignoring the optically thick cooling suppression term from Rafikov (2007)]. This increases the cooling rate at high densities substantially. However, this has no discernible effect on the simulation results, as the opacity effects on the cooling function only become important in the suppression of fragmentation at the opacity-limited mass scale $\sim 0.01 \mathrm{M}_{\odot}($ Rees 1976).

\subsubsection{Magnetic field strength}

We perform a simulation with no magnetic field and a simulation with a 'strong' magnetic field whose initial magnetic energy is equal to the initial turbulent energy, 10 times the standard value. A strong enough magnetic field may suppress fragmentation and the local SFR by as much as a factor of 2 on small scales (Federrath \& Klessen 2012), without considering feedback. We do see this effect in the 'strong' magnetic field run: the initial star formation rate is about $1 / 2$ that of the standard run. However, the SFR still continues to rise until it reaches the level set by feedback moderation, and the rest of the star formation history is quite similar to the other runs. Removing the magnetic field had no discernible effect upon the SFR, suggesting that the magnetic field has no large-scale dynamical relevance in the standard physics runs. However, we do note a smallscale cloud morphology in the MHD simulations that is distinctly more filamentary than the non-MHD simulation, due to the gas preferentially moving along magnetic field lines (see Collins et al. 2012).

\subsubsection{Slow sub-grid SFR}

In this run, we force a small-scale star formation rate $\dot{\rho}_{\star}=$ $0.01 \rho_{\mathrm{mol}} / t_{\mathrm{ff}}$ in gas that satisfies the star formation criteria (Section 3.1). This is 100 times slower than the usual choice, and comparable to the specific star formation rate on the scale of galactic discs (Kennicutt 1998b; Krumholz, Dekel \& McKee 2012). This does not affect the average SFR in our simulations because the ratelimiting step of star formation is the formation of dense, unstable gas structures in the first place. Collections of gas particles that meet the star formation criteria but have not yet turned into stars will simply continue to contract to greater densities within a local freefall time, causing the local SFR to diverge until stars inevitably form. This result is notably different from simulations which enforce the same star formation law but do not follow low-temperature cooling below $10^{4} \mathrm{~K}$ and adopt an effective equation of state for stellar feedback. In such a simulation, the local star formation law would underestimate the global star formation rate because the aforementioned gravitational contraction would be suppressed.

Note that this insensitivity to the local SFE is only obtained because the gas particle gravitational softening is fully adaptive. Otherwise, the cold gas would simply contract to inter-particle spacings 


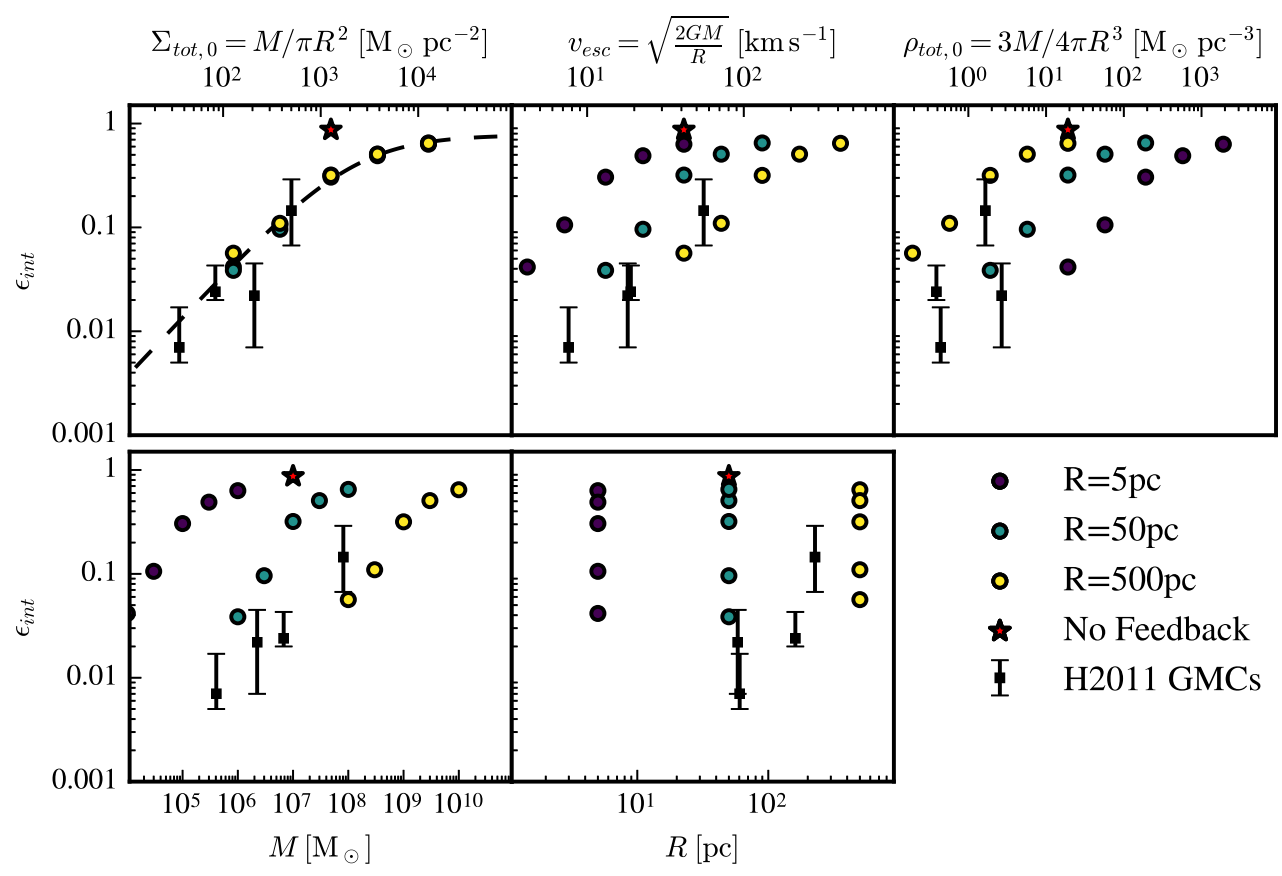

Figure 4. Integrated SFE $\epsilon_{\text {int }}$ of the 15 parameter survey simulations plotted against various functions of the initial simulation parameters $M$ (mass) and $R$ (radius). The points with error bars, 'H2011 GMCs', represent the populations of GMCs extracted from previous full-scale galaxy simulations (Hopkins et al. 2011). The points represent the population medians, and the bars represent the $\pm 1 \sigma$ percentiles. The dashed line in panel 1 is the best-fitting curves to equation (11), which gives parameters $\Sigma_{\text {crit }}=2800 \pm 100 \mathrm{M}_{\odot} \mathrm{pc}^{-2}$ and $\epsilon_{\max }=0.77 \pm 0.05$.

comparable to the minimum softening and stop at that density, and the local SFR would stop increasing.

The most notable effect of this modification was the formation of much denser and much more plentiful bound star clusters. As gas exhaustion is slowed down locally, protoclusters spend more time radiating away energy, contracting, and damping out their internal turbulent motions before turning into star particles. This increases the compactness and boundedness of the remnants. We therefore caution that while global star formation histories are not sensitive to the local value of $\epsilon_{\mathrm{ff}}$ (see also Hopkins et al. 2017), the physics of star cluster formation may be.

\subsubsection{Metallicity}

In the low-metallicity test, we scale the initial gas metallicity down from $\mathrm{Z}_{\odot}$ to $10^{-2} \mathrm{Z}_{\odot}$. This can affect many aspects of the cooling and feedback physics. Metal line cooling is proportionally less efficient, however even at $Z \sim 10^{-2} \mathrm{Z}_{\odot}, t_{\text {cool }}<<t_{\text {ff }}$ in the most dense gas, so fragmentation should not be strongly altered. This may change at metallicities of $10^{-4}-10^{-5} \mathrm{Z}_{\odot}$ (Hopkins \& Conroy 2017). The metallicity also determines dust opacity, and thus the coupling efficiency for IR radiation pressure. Lastly, it affects the evolution of the formed stellar populations' mass, energy, and momentum injection rates, which are obtained from STARBURST99. Overall, the metal-poor simulation had a SFE only marginally greater than the standard run (0.35 compared to 0.32), however it did have a faster initial growth in the SFR, suggesting that the stellar feedback at low metallicity might be less effective at halting accretion on to cluster-forming cores. The main difference in the feedback budget is due to the $\propto Z^{0.7}$ scaling of the linedriven stellar wind mass-loss rate of type O stars (Vink, de Koter \& Lamers 2001). At solar metallicity, the momentum input is somewhat less than that of radiation pressure, but the same order of mag- nitude. At $10^{-2} \mathrm{Z}_{\odot}$, however, the dynamical effect of the winds is negligible.

We have also performed limited experiments with our routines for cosmic ray heating, cooling, streaming, and diffusion. In general, if the system is given an initial cosmic ray energy density, it will rapidly cool away into dynamical irrelevance: like the magnetic field, it is ultimately a reservoir for the energies of gravitational collapse and stellar feedback, and not a source of energy in itself. There is also the possibility of the system being immersed in a strong cosmic ray background, however such environmental interactions are beyond the scope of this work. However, Yoast-Hull, Gallagher \& Zweibel (2016) have found that the cosmic ray energy in nuclear starbursts tends to be considerably smaller than the magnetic field energy, suggesting that even in the full picture with a realistic galactic environment cosmic rays should not greatly influence the overall dynamics of a collapsing GMC.

\subsection{Integrated star formation efficiency}

We now arrive at our main results. In summary, Fig. 4 the star formation efficiencies of the parameter survey simulations are plotted against the surface density, escape velocity, 3D density, mass, and radius derived from the simulation parameters $M$ and $R$. Clearly, the mass, size, density, and escape velocity are not good general predictors of $\epsilon_{\text {int }}$; similar $\epsilon_{\text {int }}$ values are obtained in simulations for which these quantities differ by orders of magnitude.

Of the obvious physical quantities derived from $M$ and $R, \Sigma_{\text {tot, } 0}$ is the best predictor of $\epsilon_{\text {int }}$, with particularly good agreement between spatial scales at high $\Sigma_{\text {tot, } 0}$, where the dynamical time is always short compared to main-sequence lifetimes. In general, we obtain good agreement with equation (9): $\epsilon_{\text {int }}$ scales $\propto \Sigma_{\text {tot, } 0}$ when $\Sigma_{\text {tot, } 0}<<\Sigma_{\text {crit }}$, and it saturates to a maximum $\epsilon_{\text {int }}$ at sufficiently high surface density. The saturation efficiency is not necessarily 1 , 
as depends on the initial conditions and what subset of the gas is used when defining $\epsilon_{\text {int }}$. As an extreme example, if the initial gas density field had an extended warm diffuse background component, as it might realistically, the diffuse gas would never form stars over the time-scale of interest, but would reduce the $\epsilon_{\text {int }}$ statistic if it were included in the gas mass sum. In our simulations, it is possible that there is a similar effect for the diffuse gas at the outer edges of the disc, as well as the gas which escapes through underdense 'chimneys' between the dense sub-clouds within the disc.

We fit $\epsilon_{\text {int }}$ to the following two-parameter model:

$\epsilon_{\text {int }}=\left(\frac{1}{\epsilon_{\max }}+\frac{\Sigma_{\mathrm{crit}}}{\Sigma_{\mathrm{tot}, 0}}\right)^{-1}$,

which is equivalent to the Fall et al. (2010) formula (equation 9) in the limit $\Sigma_{\text {tot, } 0}<<\Sigma_{\text {crit }}$ but approaches $\epsilon_{\max }$ as $\Sigma_{\text {tot }} \rightarrow \infty$. Performing an unweighted fit on $\log \epsilon_{\text {int }}$, the best-fitting parameters are $\Sigma_{\text {crit }}=2800 \pm 100 \mathrm{M}_{\odot} \mathrm{pc}^{-2}$ and $\epsilon_{\max }=0.77 \pm 0.05$. The bestfitting curve is plotted in panel 1 of Fig. 4. This value of $\Sigma_{\text {crit }}$ is within a factor of 2 of that found by Fall et al. (2010), and is compatible with the value of $\Sigma_{\text {crit }}$ found in Section 2 from the average observed $\epsilon_{\text {int }}$ of Milky Way GMCs.

The residual $R$-dependence of $\epsilon_{\text {int }}$ is small, but is positively correlated with $R$. This may be explained by the built-in scales in ISM cooling and stellar feedback physics. It is expected that the thermal pressure of the warm ISM heated to $10^{4} \mathrm{~K}$ will have a greater proportional dynamical effect in the few clouds with escape velocities that do not greatly exceed $10 \mathrm{~km} \mathrm{~s}^{-1}$. The time-scale of stellar evolution also introduces a scale into stellar feedback: at fixed $\Sigma_{\text {tot, } 0}, t_{\mathrm{ff}}$ scales $\propto R^{\frac{1}{2}}$. Therefore, as $R$ spans 2 dex, the time-scale of star formation spans an order of magnitude, so the timing of star formation relative to the stellar evolution within the formed stellar popula-

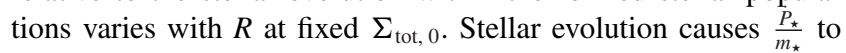
vary over time, so the effective strength of feedback that determines $\epsilon_{\text {int }}$ will be some function of the global star formation time-scale $t_{\mathrm{ff}}$. The general trend is that of increasing SFE over longer dynamical times, indicating that the effective $\frac{\dot{P}_{\star}}{m_{\star}}$ decreases monotonically with time. This is despite the increasing relevance of supernovae in the simulations spanning longer time-scales: as massive stars die, the introduction of supernovae is not enough to make up for the loss of mechanical luminosity from radiation and stellar winds to maintain the initial $\frac{\dot{P}_{\star}}{m_{\star}}$.

In Fig. 4, the compiled SFE statistics for GMC populations extracted from the parameter survey of full-scale galaxy simulations (Hopkins et al. 2012a) are also plotted for comparison, and happen to be largely compatible with the fit. In light of this and the agreement with the observational estimate of $\Sigma_{\text {crit }}$, we may safely generalize these results from our contrived generic gas ball setup to clouds with actual GMC morphologies as they emerge from galactic gas dynamics. While the large-scale morphology and relative importance of shear, rotation, and turbulence may be different between our simulations and GMCs that emerge in galaxy simulations, the scaling of $\epsilon_{\text {int }}$ is an inevitable result that applies to self-gravitating gas cloud that can form stars. Therefore, equation (11) is a general predictor of the $\epsilon_{\text {int }}$ of a star-forming gas cloud, provided that it is self-gravitating and it has some well-defined average surface density.

\subsection{Duration of star formation and per-freefall SFE}

We now discuss results concerning star formation rates and timescales. As stated in the overview, star formation in all parameter survey simulations spans no more than $\sim 3 t_{\mathrm{ff}, 0}$ (see Figs 2 and 3 ). Here, we seek to quantify this statement more precisely. As a generalpurpose measure of the duration of the starburst, we define the quantity $T_{\mathrm{SF}}$, the stellar mass formed divided by the mass-weighted average star formation rate:

$T_{\mathrm{SF}}=\frac{M_{\star}}{\left\langle\dot{M}_{\star}\right\rangle}=\frac{M_{\star}^{2}}{\int\left(\dot{M}_{\star}\right)^{2} \mathrm{~d} t}$.

This is a natural measure of characteristic of the peak in the star formation history (see Figs 2 and 3). It is also a useful proxy for the lifetime of the gas disc, as star formation largely begins once the gas has settled into a disc and halts once the disc is disrupted. The values of $T_{\mathrm{SF}}$ are tabulated in Table 2. $T_{\mathrm{SF}}$ is insensitive to the small early and late tails of the star formation history, however, so in Table 2 we also quote $T_{2 \sigma}$, the time interval containing 95 per cent of the star formation. This is generally only slightly more than $T_{\mathrm{SF}}$, as most star formation occurs in a brief burst, and feedback is able to rapidly quench star formation.

In all simulations, $T_{\mathrm{SF}} \sim t_{\mathrm{ff}, 0}$ (see Table 2 ), so most of the star formation occurs within a single initial global freefall time. This confirms our argument in Section 2: since $t_{\mathrm{ff}, 0}$ is longer than any other internal collapse time-scale, and turbulent support dissipates in a crossing time (e.g. Elmegreen 2000), the disc should be able to form enough stars to reach the blowout stage within this time. This time constraint implies a tight relation between $\epsilon_{\text {int }}$ and $\epsilon_{\mathrm{ff}}$ : if star formation is constrained to happen over $N$ dynamical times, then $\epsilon_{\mathrm{ff}}=\epsilon_{\text {int }} / N$ on average.

This brings us to a very important subtlety of feedback-moderated star formation: while stellar feedback determines $\epsilon_{\text {int }}$ in a simple way through the force balance described in Section 2, it also determines $\epsilon_{\mathrm{ff}}$ in an 'instantaneous' sense, with 'instantaneous' meaning over time-scales much longer than the dynamical time of the smallest resolved units of star formation, yet still much shorter than the global time-scale. Since star formation is a process of hierarchical fragmentation from the largest cloud scale down to individual stars, the total star formation history is the sum of a hierarchy of many individual smaller and shorter star formation events, each of which has its $\epsilon_{\text {int }}$ determined by the local ratio of feedback and gravity. This results in an overall star formation rate that is moderated 'from the bottom up'. Realistically, the 'bottom' of this hierarchy would be set by the mass scale at which it is likely that the sampled IMF contains a massive star that can exert strong feedback.

It is of limited usefulness to compare star formation time-scales to $t_{\mathrm{ff}, 0}$, at least when comparing with the value of $\epsilon_{\mathrm{ff}}$ in observed star-forming systems, as it requires knowledge of the more-diffuse initial conditions. The freefall time inferred for the gas discs as they would be observed during star formation would be something closer to $t_{\mathrm{ff}, 50}$, as derived from the mass-weighted median gas density (equation 6). ${ }^{6}$ Average values of $\epsilon_{\mathrm{ff}, 50} \equiv \dot{M}_{\star}(t) t_{\mathrm{ff}, 50}(t) / M_{\mathrm{gas}}(t)$ for each simulation can be found in columns 9 and 10 of Table 2. In panel 1 of Fig. 5 we plot $\epsilon_{\mathrm{ff}, 50}$ as a function of $\epsilon_{\text {int }}$ and confirm that there is a tight relation between two efficiencies. The best-fitting power law to the relation has an exponent within $1 \sigma$ of 1 , so we propose a simple proportional relation:

$\left\langle\epsilon_{\mathrm{ff}, 50}\right\rangle_{t}=0.34 \epsilon_{\mathrm{int}}$,

\footnotetext{
${ }^{6} \mathrm{We}$ have found that in these simulations $t_{\mathrm{ff}, 50}$ tends to be quite close to the freefall time-scale derived from the volume-averaged gas density, which is closer to what is actually calculated for GMCs. We use $t_{\mathrm{ff}, 50}$ because we have found it to be more stable and robust.
} 
Table 2. Important global quantities predicted by the simulations. Values denoted with a '+' indicate a lower bound. (1-3) As Table 1. (4) $\epsilon_{\text {int }}$, the integrated SFE (equation 3). (5) $T_{\mathrm{SF}}$, the characteristic width of the peak in the star formation history (equation 12), in Myr. (6) $T_{\mathrm{SF}}$ in units of the initial freefall time $t_{\mathrm{ff}, 0}$. (7) $T_{2 \sigma}$, the interval of time containing 95 per cent of star formation in Myr. (8) $T_{2 \sigma}$ in units of the initial freefall time $t_{\mathrm{ff}, 0}$. (9) $\left\langle\epsilon_{\mathrm{ff}, 50}\right\rangle_{t}$, the time-averaged per-freefall SFE defined in terms of the median gas density. (10) $\sigma_{\log \epsilon_{\mathrm{ff}, 50}}$, the dispersion in $\log \epsilon_{\mathrm{ff}, 50}$ in dex.

\begin{tabular}{|c|c|c|c|c|c|c|c|c|c|}
\hline \multirow{2}{*}{$\underset{\text { (1) }}{\Sigma_{\mathrm{tot}, 0}\left(\mathrm{M}_{\odot} \mathrm{pc}^{-2}\right)}$} & \multirow[b]{2}{*}{$\begin{array}{l}R(\mathrm{pc}) \\
(2)\end{array}$} & \multirow[b]{2}{*}{$\begin{array}{l}\text { Modifications } \\
\text { (3) }\end{array}$} & \multicolumn{3}{|c|}{ Global simulation results } & \multirow[b]{2}{*}{$\begin{array}{l}T_{2 \sigma}(\mathrm{Myr}) \\
\text { (7) }\end{array}$} & \multirow[b]{2}{*}{$\begin{array}{l}T_{2 \sigma} / t_{\mathrm{ff},} 0 \\
(8)\end{array}$} & \multirow[b]{2}{*}{$\begin{array}{l}\left\langle\epsilon_{\mathrm{ff}, 50}\right\rangle_{t} \\
(9)\end{array}$} & \multirow[b]{2}{*}{$\begin{array}{l}\sigma_{\log \epsilon_{\mathrm{fff}, 50}}(\operatorname{dex}) \\
(10)\end{array}$} \\
\hline & & & $\begin{array}{l}\epsilon_{\text {int }} \\
(4)\end{array}$ & $\begin{array}{l}T_{\mathrm{SF}}(\mathrm{Myr}) \\
(5)\end{array}$ & $\begin{array}{l}T_{\mathrm{SF}} / t_{\mathrm{ff}, 0} \\
(6)\end{array}$ & & & & \\
\hline 127 & 50 & & 0.04 & 7.19 & 1.23 & 8.83 & 1.51 & 0.02 & 0.56 \\
\hline 127 & 500 & & 0.06 & 25.50 & 1.38 & 35.20 & 1.90 & 0.01 & 0.55 \\
\hline 382 & 5 & & 0.11 & 0.95 & 0.89 & 1.16 & 1.09 & 0.09 & 0.70 \\
\hline 1270 & 5 & & 0.31 & 0.77 & 1.31 & 0.81 & 1.38 & 0.11 & 0.77 \\
\hline 1270 & 50 & 'Standard' & 0.32 & 2.22 & 1.20 & 2.45 & 1.32 & 0.12 & 0.79 \\
\hline 1270 & 50 & No magnetic field & 0.34 & 2.44 & 1.31 & 2.57 & 1.39 & 0.08 & 0.74 \\
\hline 1270 & 50 & Strong magnetic field & 0.30 & 2.33 & 1.26 & 2.59 & 1.40 & 0.11 & 0.66 \\
\hline 1270 & 50 & No feedback & $0.86+$ & $3.25+$ & $1.75+$ & $3.59+$ & $1.94+$ & 0.52 & 0.62 \\
\hline 1270 & 50 & Slow sub-grid SFR & 0.30 & 1.79 & 0.97 & 1.85 & 1.00 & 0.11 & 1.03 \\
\hline 1270 & 50 & $Z=10^{-2} Z_{\odot}$ & 0.35 & 2.05 & 1.11 & 2.13 & 1.15 & 0.14 & 0.75 \\
\hline 1270 & 50 & Random seeding 2 & 0.30 & 2.06 & 1.11 & 2.32 & 1.25 & 0.11 & 0.56 \\
\hline 1270 & 50 & Random seeding 3 & 0.28 & 2.03 & 1.10 & 2.23 & 1.20 & 0.10 & 0.63 \\
\hline 1270 & 50 & $150^{3}$ particle resolution & $0.26+$ & $1.98+$ & $1.07+$ & $2.12+$ & $1.15+$ & 0.10 & 0.60 \\
\hline 1270 & 50 & $50^{3}$ particle resolution & 0.33 & 2.78 & 1.50 & 3.10 & 1.67 & 0.10 & 0.37 \\
\hline 1270 & 500 & & 0.31 & 7.50 & 1.28 & 7.91 & 1.35 & 0.14 & 0.83 \\
\hline 3820 & 5 & & 0.49 & 0.55 & 1.61 & 0.61 & 1.81 & 0.13 & 0.51 \\
\hline 3820 & 50 & & 0.51 & 1.58 & 1.48 & 1.73 & 1.62 & 0.14 & 0.48 \\
\hline 3820 & 500 & & 0.50 & 5.06 & 1.50 & 5.35 & 1.58 & 0.16 & 0.50 \\
\hline 12700 & 5 & & 0.63 & 0.33 & 1.76 & 0.36 & 1.95 & 0.20 & 0.50 \\
\hline
\end{tabular}

where $\left\langle\epsilon_{\mathrm{ff}, 50}\right\rangle_{t}$ denotes the average observed value at a random point during the star formation history. The physical implication of this relation is that star formation in the simulations is indeed constrained to occur mainly within $\sim 3$ dynamical times, regardless of the relative strength of feedback and gravity, as was argued in Section 2. This would agree with the mean GMC lifetime of 3 freefall times inferred in Murray (2011).

The shape of the distribution of $\epsilon_{\mathrm{ff}}$, which we show in panel 2 of Fig. 5, is also of interest. In general, the distribution is strongly peaked near $\epsilon_{\text {int }}$, with only brief excursions above $\epsilon_{\text {int }}$. The distribution is negatively skewed due to the early and late tails of the star formation history, which spread the distribution over several orders of magnitude, similar to what is found in (Lee et al. 2016). The intrinsic dispersion in the value of $\epsilon_{\mathrm{ff}, 50}$ across the lifetime of the system (Table 2 , column 10) typically has a value between 0.4 and 0.8 dex.

\section{DISCUSSION}

\subsection{Star-forming clouds and clumps in the Milky Way}

Many star-forming clouds, identified as associations between emission from young stars and molecular gas, have been observed in the Milky Way. These clouds can be broadly classified into two groups: GMCs proper, which have characteristic surface density $100 \mathrm{M}_{\odot} \mathrm{pc}^{-2}$ and are typically traced in CO (Larson 1981; Solomon et al. 1987; Bolatto et al. 2008), and dense clumps, which have a typical surface density of $10^{3} \mathrm{M}_{\odot} \mathrm{pc}^{-2}$, and are traced in higher density tracers such as HCN (Wu et al. 2005, 2010; Heyer et al. 2016). The observational proxy of $\epsilon_{\text {int }}$ that can be obtained for these systems is

$\epsilon_{\mathrm{obs}}=\frac{M_{\star, \text { young }}}{M_{\star, \text { young }}+M_{\text {molecular }}}$,

where $M_{\star \text {, young }}$ is the mass of stars younger than $3.9 \mathrm{Myr}$, as can be traced from emission from $\mathrm{H}$ II regions or from direct counts of young stellar objects, and $M_{\text {molecular }}$ is the mass of molecular gas in the cloud. Note that both of these masses must vary during a star-forming cloud's lifetime, and in general $\epsilon_{\mathrm{obs}} \neq \epsilon_{\text {int }}$. However, the trend in $\epsilon_{\text {obs }}$ with $\Sigma_{\text {gas }}$ should still follow that of $\epsilon_{\text {int }}$, so some systematic variation in the $\epsilon_{\mathrm{obs}}$ should be evident in clouds with widely different surface densities.

In Table 3, we summarize the $\Sigma_{\text {gas }}$ and $\epsilon_{\text {obs }}$ statistics of the GMC data sets of Lee et al. (2016) and Vutisalchavakul et al. (2016) and the dense clump data sets of Wu et al. (2010) and Heyer et al. (2016). Lee et al. provides $\epsilon_{\mathrm{obs}}$ directly (denoted $\epsilon_{\mathrm{br}}$ in the paper).

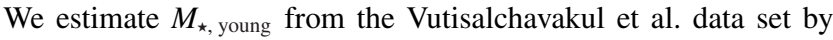
multiplying the provided SFR measurements from MIR flux by 

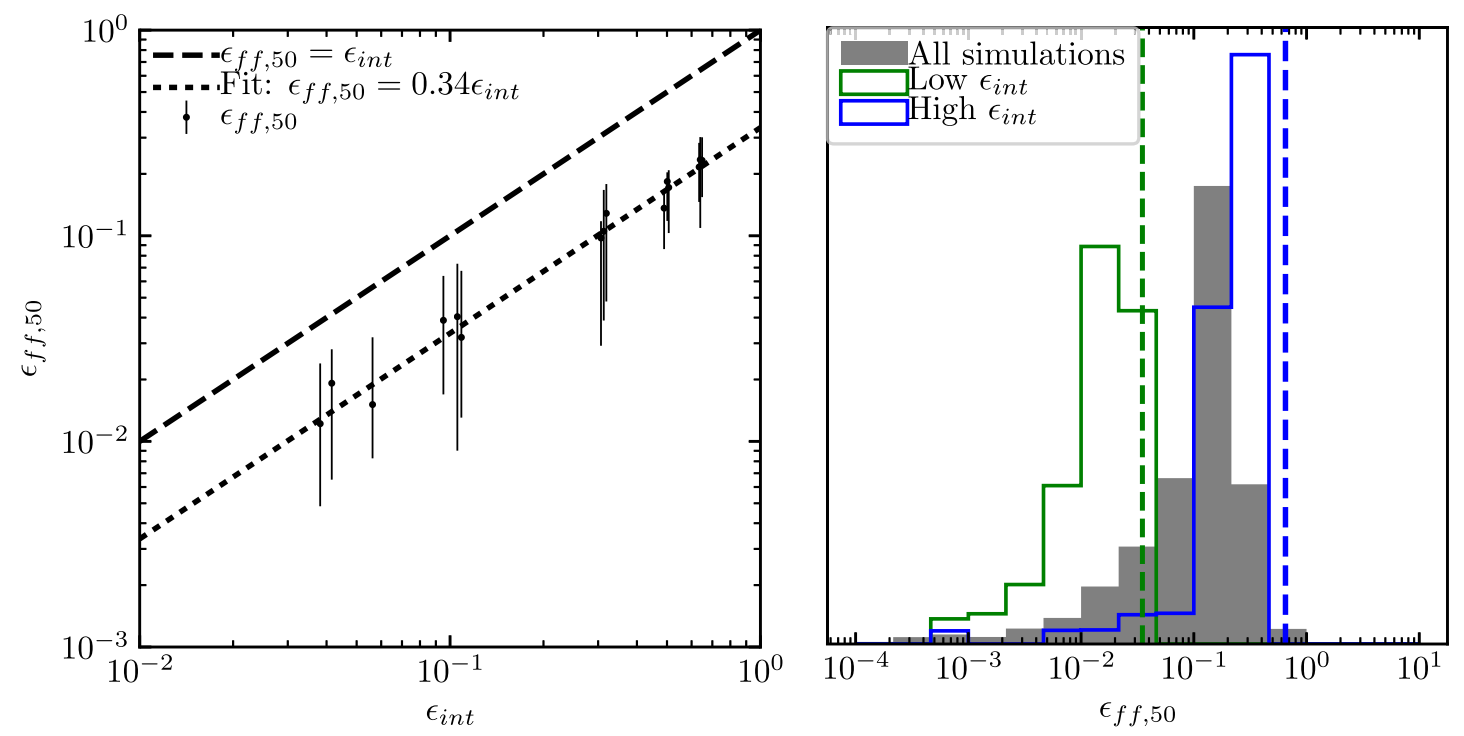

Figure 5. Left: Instantaneous per-freefall SFE $\epsilon_{\mathrm{ff}, 50}=\dot{M}_{\star}(t) t_{\mathrm{ff}, 50}(t) / M_{\mathrm{gas}}(t)$ (see equation 6) as a function of integrated SFE $\epsilon_{\text {int }}$ for all parameter survey simulations. The points represent the value of $\epsilon_{\mathrm{ff}, 50}$ averaged over all times where the SFR is non-zero. Error bars represent the $\pm 1 \sigma$ percentiles of $\epsilon_{\mathrm{ff}, 50}$. The dashed line marks the line of equality between $\epsilon_{\mathrm{ff}}$ and $\epsilon_{\mathrm{int}}$, and the dotted line indicates the best proportional fit. Right: Histograms of $\epsilon_{\mathrm{ff}, 50}$ for all parameter-survey simulations (grey), a highly efficient $\left(\epsilon_{\text {int }}=0.64\right)$ run with $\Sigma_{\text {tot }, 0}=12700 \mathrm{M}_{\odot} \mathrm{pc}^{-2}, R=50 \mathrm{pc}$ (blue), and an inefficient $\left(\epsilon_{\text {int }}=0.08\right)$ run with $\Sigma_{\mathrm{tot}, 0}=382 \mathrm{M}_{\odot} \mathrm{pc}^{-2}, R=50 \mathrm{pc}$ (green). The dashed lines indicate $\epsilon_{\text {int }}$ for the respective runs. Not surprisingly, $\epsilon_{\mathrm{ff}}, 50$ scales in proportion to $\epsilon_{\text {int }}$, but it has considerable variation $(\sim 0.4-0.8 \mathrm{dex})$ throughout the star formation history of a single simulation. For Milky Way GMCs of surface density $\sim 100 \mathrm{M}_{\odot} \odot \mathrm{pc}^{-2}$, we expect $\epsilon_{\mathrm{ff}, 50}$ to average to 0.01 , in good agreement with observations.

Table 3. Quantiles of molecular gas surface density $\Sigma_{\text {gas }}$ and the observationally inferred SFE $\epsilon_{\text {obs }}$ (equation 14) from various studies of star-forming GMCs and dense clumps in the Milky Way, in the format median ${ }_{-1 \sigma}^{+1 \sigma}$. Both $\Sigma_{\text {gas }}$ and $\epsilon_{\text {obs }}$ typically scale by $\sim 1$ dex between GMC conditions and dense clump conditions. For Heyer et al. (2016), both upper and lower bounds are provided. The final column gives the true integrated SFE $\epsilon_{\text {int }}$ predicted by substituting the median $\Sigma_{\text {gas }}$ into equation (11).

\begin{tabular}{lllll}
\hline Data set & Class & $\log \Sigma_{\text {gas }}\left(\mathrm{M}_{\odot} \mathrm{pc}^{-2}\right)$ & $\log \epsilon_{\text {obs }}$ & $\log \epsilon_{\text {int }}$ predicted from median $\Sigma_{\text {gas }}$ \\
\hline Lee et al. (2016) & GMCs & $1.88_{1.40}^{2.19}$ & $-1.97_{-2.76}^{-1.23}$ & -1.58 \\
Vutisalchavakul et al. (2016) & GMCs & $1.95_{1.68}^{2.24}$ & $-1.93_{-2.58}^{-1.37}$ & -1.51 \\
Wu et al. (2010) & Dense clumps & $3.00_{2.63}^{3.39}$ & $-1.10_{-1.76}^{-0.86}$ & -0.61 \\
Heyer et al. (2016) & Dense clumps & $2.79_{2.61}^{3.05}$ & Upper: $-0.87_{-1.29}^{-0.55}$, lower: $-2.14_{-2.71}^{-1.69}$ & -0.76 \\
\hline
\end{tabular}

3.9 Myr, the mean massive star lifetime weighted by ionizing flux

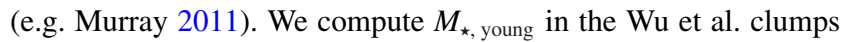
by converting the reported IR luminosities to the mass of a young single stellar population with a Kroupa (2002) IMF. In Table 3 we give values for the Heyer et al. corresponding to the value of $M_{\star \text {, young }}$ extrapolated from YSO counts assuming a Kroupa IMF (an upper bound) as well as values assuming the only mass is in stars that have been directly counted (a lower bound). As it is physically unlikely that less massive stars are not present, and the SFE from the upper bound is closer to Wu et al. (2010) and nearby star-forming regions (Lada \& Lada 2003), the true value is probably closer to the upper bound.

A $\sim 1$ dex scaling in the median $\epsilon_{\mathrm{obs}}$ is evident between $\sim 1$ per cent for the GMCs at $\sim 10^{2} \mathrm{M}_{\odot} \mathrm{pc}^{-2}$ and $\sim 10$ per cent for the clumps at $\sim 10^{3} \mathrm{M}_{\odot} \mathrm{pc}^{-2}$, in agreement with the general prediction of our SFE model. However, substituting the median surface density into our model for $\epsilon_{\text {int }}$ (equation 11) gives a SFE that is typically $\sim 0.4$ dex greater than the median $\epsilon_{\text {obs }}$. This offset could have several possible causes, including an underestimation of the strength of feedback in the simulations, the accounting of gravita- tionally bound gas in the observations, or an intrinsic bias in $\epsilon_{\mathrm{obs}}$ as an estimator of $\epsilon_{\text {int }}$.

If the scatter in the observed $\epsilon_{\text {obs }}$ were only due to intrinsic variation from the scatter in $\Sigma_{\text {gas }}$, then we would expect the scatter in $\Sigma_{\text {gas }}$ and $\epsilon_{\text {obs }}$ to be equal. This is not the case: the scatter in $\epsilon_{\text {obs }}$ is too large to be explained by the variation in $\Sigma_{\text {gas }}$ alone. This is likely due to the variation in the observed $\epsilon_{\text {obs }}$ that arises from observing the clouds at random times in their star-forming lifetimes as the stellar and molecular mass content varies (e.g. Lee et al. 2016). This type of variation is present to some extent in the simulations (e.g. Fig. 5, panel 2).

We may also compare to observational estimates of $\epsilon_{\mathrm{ff}}$. The Lee et al. and Vutisalchavakul et al. data sets give median $\epsilon_{\mathrm{ff}}$ values of $\sim 2$ per cent and $\sim 1$ per cent, respectively, which are consistent with what is found in our simulations with similar gas surface density. However, the best-fitting $\epsilon_{\mathrm{ff}}$ in dense clumps reported by Heyer et al. is also $\sim 2$ per cent when the upper bound on the stellar mass is used. In Heyer et al. (2016), the SFRs are computed by dividing the inferred stellar mass by $\tau_{\mathrm{SF}}=0.5 \mathrm{Myr}$, the evolution timescale for Class I protostars inferred from low-mass star-forming 
regions (Evans et al. 2009; Gutermuth et al. 2009). In general, inferred SFRs of dense clumps have relied on assumption that star formation has been steady for at least as long as $\tau_{\mathrm{SF}}{ }^{7}$ which is questionable within the picture presented in this paper given that nearly all clumps have freefall times shorter than this. If the lifetime of $\mathrm{HCN}$ clumps is significantly longer than $0.5 \mathrm{Myr}$ (and they are as dense as presumed) it must be due to some physics that is not accounted for in this work. One possibility is a transition in the nature of star-forming flows at lower Mach numbers, which we have hardly surveyed in our simulations. The clumps in Heyer et al. have a characteristic velocity dispersion of $\sim 0.75 \mathrm{~km} \mathrm{~s}^{-1}$, corresponding to a Mach number of 2-3, much less supersonic than GMCs at large, and in the range expected from monolithic isothermal collapse (Larson 1969; Penston 1969). Such a transition in the nature of the flow below $1 \mathrm{~km} \mathrm{~s}^{-1}$ is suggested by the inverse size-linewidth relation of clumps (Wu et al. 2010) compared to GMCs (Larson 1981). However, whether this can be responsible for reducing $\epsilon_{\mathrm{ff}}$ is unclear, as Federrath \& Klessen (2012) do not find particularly low $\epsilon_{\mathrm{ff}}$ in their $\mathcal{M}=3$ simulations. Other alternatives would include some feedback mechanism that we have not accounted for, such as protostellar heating or outflows, or a systematic overestimation of inferred density of HCN clumps (Goldsmith \& Kauffmann 2017).

Caution is needed comparing the predicted cloud lifetimes to observationally inferred lifetimes, because this is sensitive both to the observational methods/tracers, and to the actual properties (e.g. mean densities) of the initial clouds (which we have freely varied, rather than drawing from a statistically representative sample of observed clouds). A detailed comparison will be the subject of future work (Grudić et al., in preparation). However, we can make some preliminary comparisons. Lee et al. (2016) estimate a mean GMC lifetime of $\sim 24$ Myr for a population of clouds with a median free-fall time of 6.7 Myr (corresponding to a mean density of $25 \mathrm{H}_{2}$ molecules $\mathrm{cm}^{-3}$ ). Our $\Sigma=127 \mathrm{M} \odot \mathrm{pc}^{-2}, R=50 \mathrm{pc}$ run is the closest to this in mean density $\left(33 \mathrm{~cm}^{-3}\right)$ and free-fall time, and its major star formation episode lasts for $2.5 t_{\mathrm{ff}, 0} \approx 15$ Myr (see Fig. 2 and Table 1). This is somewhat smaller than observed, although similar enough that differences in how 'lifetime' is measured and observationally estimated might account for the difference. Moreover, real GMCs are not, of course, isolated, but can accrete continuously over their lifetime and may have turbulence 'stirred' externally which further can slow collapse (for a review, see Fukui \& Kawamura 2010). It seems likely therefore that clouds embedded in a realistic ISM would have somewhat longer lifetimes.

\subsection{Slow star formation}

The scaling and saturation of $\epsilon_{\mathrm{ff}}$ appears at first to be at odds with the notion of 'slow' star formation, wherein it has been observed that $\epsilon_{\mathrm{ff}} \sim 1$ per cent universally, from Milky Way like to ULIRGlike environments (Kennicutt 1998b; Krumholz \& McKee 2005; Krumholz \& Tan 2007; Krumholz et al. 2012). This slow speed of star formation has been explained theoretically in terms of the properties of the turbulent ISM alone (e.g. Krumholz \& McKee 2005; Hennebelle \& Chabrier 2011), so it is necessary to compare the predictions of these theories with those of feedback-moderated star

\footnotetext{
${ }^{7}$ The SFRs of the Wu et al. (2010) HCN clumps, as determined by Heiderman et al. (2010) from infrared luminosity, have $\epsilon_{\mathrm{ff}} \sim 1$ per cent, but again there is an implicit averaging window $\tau_{\mathrm{SF}} \sim 4 \mathrm{Myr}$ in the $L_{\mathrm{IR}}-\mathrm{SFR}$ conversion factor used. This figure of 1 per cent does appear to be a general finding for dense clumps (Krumholz 2014).
}

formation to determine whether feedback is a necessary part of the picture. In making this comparison, we emphasize that our prediction pertains to individual unstable clouds near virial equilibrium, and not to any significant patch of a galaxy that may contain GMCs in various states of formation and disruption, as well as the other phases of the ISM. In the latter case, it has been shown in Hopkins et al. (2014) and Orr et al. (2017) that the same physical models used in our simulations also robustly predict that $\epsilon_{\mathrm{ff}, \text { gal }} \sim 1$ per cent on galactic scales on average, despite assuming that $\epsilon_{\mathrm{ff}}=1$ on the smallest resolvable scales, as star formation reaches a statistical equilibrium when smoothed on $>1 \mathrm{kpc}$ scales.

Both the feedback-disrupted cloud picture suggested by our simulations and purely turbulence-regulated star formation theories successfully predict the median value $\epsilon_{\mathrm{ff}} \sim 1$ percent in Milky Way GMCs, however they do so for completely different physical reasons. However, the observed dispersion in $\epsilon_{\mathrm{ff}}$ for a given set of cloud conditions has not been found to be less than 0.5 dex (Heiderman et al. 2010; Evans, Heiderman \& Vutisalchavakul 2014; Heyer et al. 2016; Lee, Miville-Deschênes \& Murray 2016; Vutisalchavakul et al. 2016); Lee et al. found 0.91 dex from the most complete Milky Way GMC data set that we are aware of. As they noted, the turbulence-regulated models do not predict this much scatter because they do not allow for $\epsilon_{\mathrm{ff}}$ to vary for a given set of turbulent ISM conditions. Lee et al. showed that the scatter can arise from observing GMCs at random points in their lifetime of initial collapse, star formation, and feedback disruption. For Milky Way like conditions, our simulations do predict intrinsic dispersions in $\epsilon_{\mathrm{ff}}$ of the same order as what has been observed; whether the figure of 0.91 dex can be fully accounted for depends upon the relationship between $\epsilon_{\mathrm{ff}}$ and its observational proxy, which we will address in future work.

The gas-rich nuclei in Arp 220 provide an interesting case study for the speed of star formation. The total SFR of $240 \mathrm{M}_{\odot} \mathrm{yr}^{-1}$, inferred from its IR luminosity (Downes \& Solomon 1998; Kennicutt 1998a), appears to agree nicely with the theory of slow star formation, yet our simulations at comparable gas surface density $\sim 10^{4} \mathrm{M}_{\odot} \mathrm{pc}^{-2}$ predict $\epsilon_{\mathrm{ff}} \sim 20$ per cent. Considering several $10^{9} \mathrm{M}_{\odot}$ of gas localized within two discs, each with radius smaller than 100 pc (Scoville et al. 2017), the resulting SFR should be well in excess of $10^{3} \mathrm{M}_{\odot} \mathrm{yr}^{-1}$, an order of magnitude greater than the $L_{\mathrm{IR}}$-inferred value. Our simulations do not consider the stabilization of the gas disc due to the presence of the central SMBH, but this can probably only reduce the predicted SFR by a factor of a few (Utreras, Becerra \& Escala 2016). The apparent discrepancy may lie in the use of $L_{\mathrm{IR}}$ to determine the SFR, as it only provides an average value over the lifetime of OB stars, 4 Myr. Because the dynamical time in the nuclear discs is of order $10^{5} \mathrm{yr}$ (Scoville et al. 2017), it is unlikely that the SFR has been steady over this comparatively long averaging window. Estimates of the SFR from supernova rates have the same limitation. Therefore, the possibility that the SFR in Arp 220 has recently been in excess of $10^{3} \mathrm{M}_{\odot} \mathrm{yr}^{-1}$ cannot be excluded on this basis (Anantharamaiah et al. 2000; Parra et al. 2007).

\subsection{Comparison with other GMC star formation studies}

Many numerical studies have been performed that are conceptually similar to the ones in this paper, following the collapse of an idealized turbulent cloud and the resulting star formation and feedback processes. It is useful to compare and contrast our predictions with these studies, in particular in cases where specific feedback mechanisms have been considered in greater detail. 
Our run without stellar feedback is most comparable with previous simulations of isothermal supersonic MHD turbulence with gravity (Kritsuk, Norman \& Wagner 2011; Collins et al. 2012; Padoan, Haugbølle \& Nordlund 2012; Lee, Chang \& Murray 2015). In these simulations, the SFR tends to grow until $\epsilon_{\mathrm{ff}}$ is of order unity, with its particular value depending somewhat upon the regular and Alfvénic Mach numbers, the virial parameter, and the details of the turbulent driving, and the final $\epsilon_{\text {int }} \sim 1$ due to the lack of feedback. The value $\epsilon_{\mathrm{ff}}=0.52$ we obtain in feedback-free cloud collapse without feedback is most consistent with the Federrath \& Klessen (2012) models with mixed or solenoidal driving.

Dale et al. (2012) ran a parameter study of feedback-disrupted clouds, considering only photoionization heating. We have found in tests that photoionization heating only is insufficient to disrupt a cloud with an escape velocity that is large compared to the sound speed $c_{\mathrm{s}} \sim 10 \mathrm{~km} \mathrm{~s}^{-1}$ of photoionized gas. This agrees with the trend of Dale et al. (2012), which found order-unity $\epsilon_{\text {int }}$ in clouds with high escape velocity (Runs ' $\mathrm{X}$ ' and ' $\mathrm{F}$ '). Also, our $M=10^{4} \mathrm{M}_{\odot} \mathrm{pc}^{-2}$, $R=5$ pc has the same physical parameters as Run ' $\mathrm{J}$ ' in Dale et al. (2012). This had $\epsilon_{\text {int }}=0.04$, while the final stellar mass in Run ' $J$ ' was 35 per cent and rising at 3.5 Myr. We re-simulated this run with photoionization heating only and radiation pressure only, and the one with photoionization heating had a very similar star formation history and cloud morphology to Run ' $\mathrm{J}$ '. The one with radiation pressure only had $\epsilon_{\text {int }}=0.05$, very close to the full physics run. Radiation pressure is thus the primary feedback mechanism even in this region of parameter space where photoionization heating alone could still theoretically disrupt the cloud.

The radiation hydrodynamics star formation simulations of Raskutti, Ostriker \& Skinner (2016) focus upon the effects of stellar feedback from the single-scattered monochromatic photons at a high opacity corresponding to UV photons. They use the radiation hydrodynamics code HYPERION, evolving the radiation field on a fixed grid according to the M1 closure (Skinner \& Ostriker 2013). They overpredict the efficiency of their fiducial Milky Way like GMC run by an order of magnitude, obtaining $\epsilon_{\text {int }}=0.43$ for a cloud with $M=5 \times 10^{4} \mathrm{M}_{\odot}$ and $R=15 \mathrm{pc}$, which has average surface density $70 \mathrm{M}_{\odot} \mathrm{pc}^{-2}$. Extrapolating our simulation results using equation (11) gives $\epsilon_{\text {int }}=0.02$ for a cloud with these parameters, in much better agreement with observations (Section 5.1 and references therein). We have found that $\epsilon_{\text {int }} \sim 0.04$ in a test run with otherwise similar initial conditions to Raskutti et al. and radiation pressure as the only feedback (Appendix A2).

This order of magnitude discrepancy may be due to the behaviour of the M1 closure in such an optically thick, multisource radiative transfer problem. Experiments in developing GIZMO's own M1 RHD scheme have shown that the momentum imparted to the gas by the radiation field around an embedded source can be underestimated by an order of magnitude if the attenuation length $\lambda=\rho^{-1} \kappa_{\mathrm{UV}}^{-1}$ is not well-resolved, which it certainly is not at the densities, opacities, and spatial resolution typical in the Raskutti et al. simulations. ${ }^{8}$ Secondly, photons propagated via the M1 scheme behave collisionally: colliding streams will form a shock rather than passing through each other. As stars form in a tightly clustered configuration in isothermal fragmentation (Guszejnov, Hopkins \& Krumholz 2017; Guszejnov, Hopkins \& Grudić 2017), neighbouring stars particles can cancel

${ }^{8}$ This problem is averted by the shell-driving test problem presented by Raskutti et al., because the radiation first propagates through an optically thin medium where the field is well-resolved. each other's fluxes. In summary, it is reasonable to suspect that ability of radiation pressure to disrupt the GMC was underestimated.

Tsang \& Milosavljevic (2017) simulated super star cluster formation in cloud with mass $10^{7} \mathrm{M}_{\odot}$ and diameter $25 \mathrm{pc}$, for a mean surface density of $1.6 \times 10^{4} \mathrm{M}_{\odot} \mathrm{pc}^{-2}$, comparable to the densest runs in our parameter study. They accounted for feedback via infrared radiation pressure, which is expected to dominate, with an accelerated Monte Carlo scheme that is more realistic than our more approximate treatment. They found that radiation pressure reduced $\epsilon_{\text {int }}$ by $\sim 30$ per cent compared to the run with no feedback. Our simulations at this surface density had $\epsilon_{\text {int }} \sim 0.64$, compared to $0.86+$ with no feedback, so despite our different treatments of radiation pressure the agreement is quite good.

It should be noted that most star formation in all simulations mentioned in this subsection occurs within some small $(\sim 2-3)$ number of global freefall times, regardless of the final $\epsilon_{\text {int }}$ if the cloud is disrupted. This naturally leads to the linear relation between $\epsilon_{\text {int }}$ and $\epsilon_{\mathrm{ff}}$ shown in Section 4.3, suggesting that this is a very general feature of the star formation-cloud disruption process, insensitive to the details of stellar feedback. The role of feedback on cloud scales is to make star formation less efficient in a given amount of time, not to prolong the star-forming lifetime as it does on galactic scales.

\subsection{Bound star cluster formation}

$\epsilon_{\text {int }}$ should be an important quantity for the formation of bound star clusters. If all other factors are equal, the fraction of a star cluster remaining gravitationally bound after gas expulsion should increase with $\epsilon_{\text {int }}$ (Tutukov 1978; Hills 1980; Mathieu 1983; Lada, Margulis \& Dearborn 1984; Elmegreen \& Clemens 1985). ${ }^{9}$ It can thus be argued that the bound cluster formation efficiency $\Gamma$, the fraction of stars found in bound clusters, is a function of $\epsilon_{\text {int }}$, and hence of $\Sigma_{\text {tot, } 0}$ by equation (9). If equation (9) holds, then cluster formation should be generic to regions of high $\Sigma_{\text {gas }}$. And indeed, rich populations of young bound clusters are ubiquitous in dense nuclear starbursts, including notable examples Arp 220 (Wilson et al. 2006), M82 (McCrady \& Graham 2007), and M83 (Bastian et al. 2012; Ryon et al. 2015). However, whether there actually is a general scaling in $\Gamma$ that depends on a single environmental parameter associated with surface density is currently an open problem: Adamo et al. (2015) and Johnson et al. (2016) appear to support this hypothesis, while Chandar, Fall \& Whitmore (2015) does not; correlations between $\Sigma_{\text {gas }}{ }^{10}$ and $\Gamma$ are apparent, but whether they are universal has not been established conclusively (Adamo et al. 2017).

GMCs in the Milky Way and other nearby galaxies typically have $\Sigma_{\text {gas }} \sim 100 \mathrm{M}_{\odot} \mathrm{pc}^{-2}$ (Larson 1981; Solomon et al. 1987), giving $\epsilon_{\text {int }} \sim 3$ percent at best, yet young bound star clusters are still observed to have formed within the galaxy (Portegies Zwart, McMillan \& Gieles 2010). Rather than simply turning off below a certain surface density threshold, $\Gamma$ is theoretically expected to scale smoothly as a function of $\Sigma_{\text {gas }}$, saturating to a value of $\sim 70$ per cent (Kruijssen 2012). Star cluster formation may be possible in environments that are less dense on average because star-forming clouds are hierarchically structured, with a broad surface density PDF. If $\epsilon_{\text {int }}$ is determined in a scale-free fashion according to equation (9), it will apply just as well on the scale of denser-than-average sub-clumps

\footnotetext{
${ }^{9}$ Other factors influencing the bound fraction of a cluster include the virial state of the stars at gas expulsion Goodwin (2009) and the degree of initial degree of clumpy sub-structure (Smith et al. 2011, 2013).

${ }^{10}$ Or equivalently $\Sigma_{\text {SFR }}$ (e.g. Kennicutt 1998b).
} 
once they decouple from their environment, allowing them to have high $\epsilon_{\text {int }}$ locally even if $\epsilon_{\text {int }}$ is small on larger scales (e.g. Kruijssen et al. 2012). If this argument is valid, we expect to see some amount of bound cluster formation in any star-forming environment.

The production of bound star clusters is generally associated with high-pressure environments, where the pressure associated with the midplane of a galactic disc can be estimated as $P \sim G \Sigma_{\text {gas }} \Sigma_{\text {tot }}$ (Elmegreen \& Efremov 1997). Elmegreen \& Efremov proposed a picture wherein GMCs are confined by this pressure $P \sim \rho v_{t}^{2}$, rather than their self-gravity, and the gas mass-loss rate in a protocluster was assumed to be $\dot{M} \propto L / v_{t}^{2}$, where $L$ is the protocluster luminosity. Thus, the fraction of the gas mass converted to stars with fixed $\epsilon_{\mathrm{ff}}$ is greater when $P$ is greater. The picture suggested by the simulations in this paper is presents an alternative to this; $\epsilon_{\mathrm{ff}}$ is not fixed, and the time-scale of mass-loss is always of the order of the freefall time. Clouds are confined by self-gravity, rather than external pressure, and their SFE is greater at greater $P \sim G \Sigma_{\text {gas }}^{2}$ because of the relative scaling of the strength of feedback and self-gravity.

In future work we will use these simulations to study the mapping between galactic environments and the populations of bound star clusters they produce, providing the stepping stone between lower resolution cosmological simulations and single-cluster dynamical studies. This development is necessary, in particular, for the theory of cosmological SMBH seed formation from runaway stellar mergers in dense clusters (see Mouri \& Taniguchi 2002; Portegies Zwart \& McMillan 2002; Gürkan, Freitag \& Rasio 2004; Devecchi \& Volonteri 2009). It would also allow a more self-consistent model of pairing and evolution of the population of massive $\left(\sim 60 \mathrm{M}_{\odot}\right)$ black hole binaries like the progenitor of GW150914 (Abbott et al. 2016); a significant fraction of these are expected to be found in bound star clusters (Rodriguez et al. 2015; Rodriguez, Chatterjee \& Rasio 2016).

\subsection{The nature of nuclear star formation}

Our results here illustrate the claim of Torrey et al. (2017): no equilibrium exists for gas-rich nuclear discs with short dynamical times, and their dynamics have an inherently transient nature: they undergo rapid fragmentation followed by rapid gas expulsion. Star-forming nuclear disc calculations must account for stellar feedback in a way that is appropriate to their short time-scales, or else risk obtaining unphysical solutions. This caveat may very well limit the validity of isolated nuclear disc simulations that use a Springel \& Hernquist (2003)-like effective EOS ISM model and a slow subgrid star formation law, both of which have been widely used in the field of galaxy simulations. For example, Hopkins \& Quataert (2010) simulated circumnuclear discs of similar mass and radius to the ones in this paper, but in absence of the appropriate feedback physics the SFR of the discs was quite likely underestimated by at least an order of magnitude.

A robust result of our simulations is that both $\epsilon_{\text {int }}$ and $\epsilon_{\mathrm{ff}}$ must saturate to $\sim 1$ at surface densities in excess of $10^{4} \mathrm{M}_{\odot} \mathrm{pc}^{-2}$. Barring other unaccounted-for feedback physics (see Section 5.7), and neglecting environmental interactions, we conclude that a gasdominated cloud with $\Sigma_{\text {gas }} \gg 10^{3} \mathrm{M}_{\odot} \mathrm{pc}^{-2}$ will convert nearly all of its gas to stars in a few crossing times. In this limit, we expect a result similar to our simulations: a population of massive star clusters will form, and will eventually merge into a single cluster because the high global SFE will allow the system to remain bound. If a relatively low-mass $\mathrm{SMBH}$ is present, it may sink to the centre of this cluster under dynamical friction. However, it is also possible that before the final nuclear cluster has formed, the $\mathrm{SMBH}$ and clusters effectively behave as a few- $N$-body system, which has chaotic behaviour and often results in the ejection of one or more members. Such ejections will prolong the time necessary for SMBH to form binary pairs in galaxy mergers, and may lower the resulting low-frequency gravitational wave background.

If star formation occurs near an SMBH, the gravity of the SMBH also contributes to the binding force on the gas. If we re-derive 9 and consider only the force of gravity of the SMBH on the gas, we obtain a lower bound for the integrated SFE of a gas disc of radius $R$ around a black hole of mass $M_{\mathrm{BH}}$ :

$\epsilon_{\text {int }} \geq\left(1+\frac{\pi R^{2} \Sigma_{\text {crit }}}{M_{\mathrm{BH}}}\right)^{-1}$.

This assumes that the gas is not somehow being prevented from forming stars by AGN feedback and that the dynamical effect of the black hole upon the gas flow does not slow star formation enough to make the gas consumption time longer than $\sim 10 \mathrm{Myr}$. The characteristic radius at which $\epsilon_{\text {int }}$ saturates to $\sim 1$ is then

$R_{\mathrm{SF}} \sim \sqrt{M_{\mathrm{BH}} / 2 \pi \Sigma_{\text {crit }}}=6 \mathrm{pc}\left(\frac{M_{\mathrm{BH}}}{10^{6} \mathrm{M}_{\odot}}\right)^{\frac{1}{2}}$,

using $\Sigma_{\text {crit }}=2800 \mathrm{M}_{\odot} \mathrm{pc}^{-2}$.

Under these assumptions, the in situ formation of a nuclear star cluster could proceed as follows: if enough low-angular momentum gas falls within $R_{\mathrm{SF}}$ of an $\mathrm{SMBH}$ to become gravitationally unstable, it will be rapidly consumed by star formation, leaving behind a nuclear star cluster and little remaining gas. The fiducial value $6 \mathrm{pc}$ derived here does lie in the range of effective radii of nuclear star clusters found in several different types of galaxies (see Hopkins et al. 2010, and references therein).

Such efficient star formation near black holes may have drastic implications for the ability of gas from the galactic disc to be accreted on to a central SMBH, as the gas may fragment into stars before reaching the hole within a few dynamical times, at which point it can no longer lose angular momentum efficiently. This contrasts greatly with models which assume star formation must be slow $\left(\epsilon_{\mathrm{ff}} \sim 1\right.$ percent) all the way down to the black hole; in this case, a steady supply of gas can reach the black hole even with modest torques, as gas has $\sim 100$ dynamical times to lose its angular momentum before being converted to stars. As such, it is important that studies of AGN accretion on $\sim \mathrm{pc}$ and smaller scales consider the physics of the multiphase ISM and star formation in some detail.

\subsection{Absence of metal-enriched supermassive direct-collapse objects}

These simulations were originally conceived as an attempt to reproduce the mechanism for direct-collapse supermassive black hole formation simulated in Mayer et al. $(2010,2015)$ with a more realistic approach to cooling and star formation. To summarize, these works propose that in the gas-rich nuclear disc resulting from a galaxy merger, fragmentation can be suppressed by some combination of turbulence and suppression of cooling due to optical thickness, enabling accretion on to a supermassive quasi-star even for ISM with solar metal abundances. To avoid overcooling in optically thick regions, we implemented the optically thick cooling approximation of Rafikov (2007) so as to interpolate between the optically thin and thick cooling regimes where appropriate. In previous tests we also chose a rather high $\left(10^{7} \mathrm{~cm}^{-3}\right)$ density threshold for star formation and allowed star formation only when the local 
Jeans mass is $<10^{3} \mathrm{M}_{\odot}$, so as to prevent premature conversion of gas particles into star particles where they may otherwise form a supermassive object. Our simulations reach comparable optical depths and turbulent velocity dispersions to the nuclear discs in the Mayer simulations, however we report no formation of directcollapse objects. In numerical experiments, we have only been able to produce anything resembling a supermassive quasi-star if we implement a temperature floor of $10^{4} \mathrm{~K}$ and slow the local star formation rate $\dot{\rho}_{\star}$ to 1 per cent of the usual value. As these are similar to the choices made for Mayer et al. $(2010,2015)$, it seems that metalenriched direct-collapse object formation is a numerical artefact of slow sub-grid star formation and a lack of low-temperature cooling. Our conclusions agree with those obtained by Ferrara, Haardt \& Salvaterra (2013) using a one-dimensional disc model: if realistic low-temperature cooling is accounted for, the cooling time in the metal-enriched ISM is invariably too short to suppress fragmentation down to the scales required to directly form a supermassive object.

\subsection{Feedback physics uncertainties}

Most of what is known about the effects of stellar feedback on GMC scales has been learned from observations of star-forming complexes within the Milky Way, and even then the true efficiencies of many feedback mechanisms acting in Milky Way like environments are still loosely constrained, to say nothing of generalizing these mechanisms to ULIRG-like environments. Here, we list uncertainties in the strength of feedback which could conceivably affect our results.

\subsubsection{The initial mass function}

Throughout this work, we have assumed that the initial stellar mass function, and hence $\dot{P}_{\star} / m_{\star}$, is independent of the environment of star formation. If the IMF were to become more top-heavy in environments of high surface density, $\dot{p}_{\star} / m_{\star}$ would increase, and as our simulations have shown, this is the quantity to which our results are most sensitive. Supposing that $\frac{\dot{P}_{\star}}{m_{\star}}$ did scale at least linearly with $\Sigma_{\text {gas }}$ due to enhanced type O star production, this would limit the maximum SFE. There is some observational evidence of a dearth of low-mass stars in dense nuclear environments (Smith \& Gallagher 2001; Bartko et al. 2010), however such observations can be subject to significant sampling bias because the time-scale for mass segregation is short in dense systems. For this reason and others, Bastian, Covey \& Meyer (2010) concluded that current observations were still largely consistent with a universal IMF.

\subsubsection{Infrared radiation pressure}

Radiation pressure plays an important role in the feedback budget in many of our simulations; even in cases where the final gas blowout is ultimately due to $\mathrm{SNe}$, radiation helps prevent an initial runaway of the SFE before SNe start to occur. We have found that $\epsilon_{\text {int }}$ saturates to a value close to 1 as surface density becomes large, however Murray et al. (2010) argued that the IR opacity of dust grains should limit the saturation point of $\epsilon_{\text {int }}$ for gas with solar abundances, as radiation pressure in the optically thick regime is the only force of feedback which can conceivably scale as fast as the gas selfgravity. By this argument, the saturation $\operatorname{SFE} \epsilon_{\mathrm{int}}^{\max }$ is expected to scale $\sim\left(\kappa_{\text {IR }} \Sigma_{\text {crit }}\right)^{-1}$, which takes a value of $\sim \frac{1}{2}$ for gas with solar metal abundances. However, in a realistic, three-dimensional scenario where hydrodynamics is coupled to the radiation field in an inhomogeneous ISM, it is actually unlikely that radiation pressure can achieve the whole ' $\tau_{\text {IR }}$ boost', either because photons will have a tendency to leak out of the most optically thin lines of sight, or because the radiative Rayleigh-Taylor instability is able to efficiently dissipate kinetic energy (Krumholz \& Thompson 2012). Radiation hydrodynamics studies on this problem are ongoing (see also Krumholz \& Thompson 2013; Davis et al. 2014; Rosdahl \& Teyssier 2015; Skinner \& Ostriker 2015; Tsang \& Milosavljević 2015; Zhang \& Davis 2017), and although results have varied with the radiative transfer scheme used, they do generally agree that the scaling of the momentum deposited to the gas with the mean $\tau_{\mathrm{IR}}$ is sub-linear for sufficiently large $\tau_{\mathrm{IR}}$, forcing the integrated SFE to ultimately saturate to $\sim 1$.

\section{SUMMARY}

We have performed a parameter study of 3D multiphysics MHD simulations of star-forming gas discs with initial parameters spanning two orders of magnitude in surface density and in spatial scale, including the physics of supernovae, stellar winds, radiation pressure, and photoionization heating. Due to the generality of the simulation setup, we have been able to study the nature of star formation in gas-rich environments in general, including nuclear starbursts and GMCs. Our main findings are as follows:

(i) In any bound, gas-rich star-forming cloud with short $(\sim 10$ Myr or less) dynamical time, star formation proceeds until it causes an inevitable gas blowout, with the final SFE determined mainly by the balance of feedback and gravitation, with other physical mechanisms having secondary importance.

(ii) The integrated SFE $\epsilon_{\text {int }}$ of such a system scales strongly with the initial gas surface density $\Sigma_{\text {tot, } 0}$ with weak dependence upon other parameters, and saturates to a value $\sim 1$ at adequately high surface density, despite the effects of strong feedback. We find good agreement with analytic derivations of $\epsilon_{\text {int }}$ which take the form of equation (9) (Fall et al. 2010; Murray et al. 2010; Dekel \& Krumholz 2013; Thompson \& Krumholz 2016), fitting a value $\Sigma_{\text {crit }}=2800 \mathrm{M}_{\odot} \mathrm{pc}^{-2}$ from the simulations. The agreement across different spatial scales is non-trivial and somewhat surprising, as our parameter space bridges distinct time-scale regions where radiation pressure (<3 Myr) and SN explosions ( $>3 \mathrm{Myr}$ ) dominate the feedback energy and momentum budget. The prediction of this SFE model is that $\epsilon_{\text {int }}$ in self-gravitating clouds should scale from $\sim 1$ per cent at $10^{2} \mathrm{M}_{\odot} \mathrm{pc}^{-2}$ and $\sim 10$ per cent at $10^{3} \mathrm{M}_{\odot} \mathrm{pc}^{-2}$, as is found in local GMCs and dense clumps (Section 5.1 and references therein). The model also predicts that SFE ultimately saturates to $\sim 100$ per cent in the limit of very high surface density.

(iii) We find a proportional relation between the integrated SFE $\epsilon_{\text {int }}$ and the per-freefall SFE $\epsilon_{\text {ff }}$ (equation 13) for self-gravitating clouds, essentially because the clouds always produce enough stars to self-destruct within $\sim 2-3$ dynamical times. $\epsilon_{\mathrm{ff}}$ is determined only initially by such details as cooling and magnetic fields, and will inevitably grow until moderated by stellar feedback. The observed $\epsilon_{\mathrm{ff}}$ distribution for Milky Way GMCs can be accounted for by combining the spread from this relation and a modest intrinsic spread due to the time-varying SFE of a single cloud. The variation in $\epsilon_{\mathrm{ff}}$ is at odds with a universal slow star formation $\left(\epsilon_{\mathrm{ff}} \sim 1\right.$ per cent $)$ law when applied to individual clouds, but the same physics used in this study recover the $\epsilon_{\mathrm{ff}, \text { gal }} \sim 1$ per cent relation in cosmological simulations (Hopkins et al. 2014, 2017).

Thus, we have determined the basic properties of feedbackmoderated star formation for self-gravitating, unstable gas 
complexes. In a subsequent paper, we have used these simulations to study the process of star cluster assembly (Grudić et al. 2017). Future work will elucidate the relation between theoretical predictions of cloud SFE and its observational proxies, the mapping between galactic environmental properties and populations of star clusters, and the detailed dynamical history of star cluster formation as determined by feedback.

\section{ACKNOWLEDGEMENTS}

We thank Neal J. Evans II, Eve Ostriker, Dávid Guszejnov, Chris Hayward, Matthew Orr and Andrew Wetzel for helpful comments and critique. We also thank the anonymous referees for highly comprehensive and helpful feedback that motivated a more thorough understanding of our results. Support for PFH and MYG was provided by an Alfred P. Sloan Research Fellowship, NASA ATP Grant NNX14AH35G and NSF Collaborative Research Grant \#1411920 and CAREER grant \#1455342. Numerical calculations were run on the Caltech computer cluster 'Zwicky' (NSF MRI award \#PHY0960291) and allocation TG-AST130039 granted by the Extreme Science and Engineering Discovery Environment (XSEDE) supported by the NSF.

\section{REFERENCES}

Abbott B. P. et al., 2016, Phys. Rev. Lett., 116, 061102

Adamo A., Kruijssen J. M. D., Bastian N., Silva-Villa E., Ryon J., 2015, MNRAS, 452, 246

Adamo A. et al., 2017, ApJ, 841, 131

Agertz O., Kravtsov A. V., Leitner S. N., Gnedin N. Y., 2013, ApJ, 770, 25 Anantharamaiah K. R., Viallefond F., Mohan N. R., Goss W. M., Zhao J. H., 2000, ApJ, 537, 613

Bartko H. et al., 2010, ApJ, 708, 834

Bastian N., Covey K. R., Meyer M. R., 2010, ARA\&A, 48, 339

Bastian N. et al., 2012, MNRAS, 419, 2606

Bate M. R., Bonnell I. A., Price N. M., 1995, MNRAS, 277, 362

Bolatto A. D., Leroy A. K., Rosolowsky E., Walter F., Blitz L., 2008, ApJ, 686, 948

Bryant P. M., Scoville N. Z., 1999, AJ, 117, 2632

Chandar R., Fall S. M., Whitmore B. C., 2015, ApJ, 810, 1

Collins D. C., Kritsuk A. G., Padoan P., Li H., Xu H., Ustyugov S. D., Norman M. L., 2012, ApJ, 750, 13

Dale J. E., Ercolano B., Bonnell I. A., 2012, MNRAS, 424, 377

Davis S. W., Jiang Y.-F., Stone J. M., Murray N., 2014, ApJ, 796, 107

Dekel A., Krumholz M. R., 2013, MNRAS, 432, 455

Devecchi B., Volonteri M., 2009, ApJ, 694, 302

Downes D., Solomon P. M., 1998, ApJ, 507, 615

Elmegreen B. G., 1983, MNRAS, 203, 1011

Elmegreen B. G., 2000, ApJ, 530, 277

Elmegreen B. G., 2007, ApJ, 668, 1064

Elmegreen B. G., Clemens C., 1985, ApJ, 294, 523

Elmegreen B. G., Efremov Y. N., 1997, ApJ, 480, 235

Evans N. J., II, et al., 2009, ApJS, 181, 321

Evans N. J., II, Heiderman A., Vutisalchavakul N., 2014, ApJ, 782, 114

Fall S. M., Krumholz M. R., Matzner C. D., 2010, ApJ, 710, L142

Faucher-Giguère C.-A., Quataert E., Hopkins P. F., 2013, MNRAS, 433, 1970

Federrath C., Klessen R. S., 2012, ApJ, 761, 156

Federrath C., Schober J., Bovino S., Schleicher D. R. G., 2014, ApJ, 797, L19

Ferrara A., Haardt F., Salvaterra R., 2013, MNRAS, 434, 2600

Freeman P., Rosolowsky E., Kruijssen J. M. D., Bastian N., Adamo A., 2017, MNRAS, 468, 1769

Fukui Y., Kawamura A., 2010, ARA\&A, 48, 547

Goldsmith P. F., Kauffmann J., 2017, ApJ, 841, 25
Goodwin S. P., 2009, Ap\&SS, 324, 259

Grudić M. Y., Guszejnov D., Hopkins P. F., Lamberts A., Boylan-Kolchin M., Murray N., Schmitz D., 2017, MNRAS, preprint (arXiv:1708.09065) Gürkan M. A., Freitag M., Rasio F. A., 2004, ApJ, 604, 632

Guszejnov D., Hopkins P. F., Krumholz M. R., 2017, MNRAS, 468, 4093

Guszejnov D., Hopkins P. F., Grudić M. Y., 2017, MNRAS, preprint (arXiv:1707.05799)

Gutermuth R. A., Megeath S. T., Myers P. C., Allen L. E., Pipher J. L., Fazio G. G., 2009, ApJS, 184, 18

Heiderman A., Evans N. J., II, Allen L. E., Huard T., Heyer M., 2010, ApJ, 723,1019

Hennebelle P., Chabrier G., 2011, ApJ, 743, L29

Heyer M., Gutermuth R., Urquhart J. S., Csengeri T., Wienen M., Leurini S., Menten K., Wyrowski F., 2016, A\&A, 588, A29

Hills J. G., 1980, ApJ, 235, 986

Hollyhead K., Bastian N., Adamo A., Silva-Villa E., Dale J., Ryon J. E., Gazak Z., 2015, MNRAS, 449, 1106

Hopkins P. F., 2015, MNRAS, 450, 53

Hopkins P. F., Conroy C., 2017, ApJ, 835, 154

Hopkins P. F., Quataert E., 2010, MNRAS, 407, 1529

Hopkins P. F., Raives M. J., 2016, MNRAS, 455, 51

Hopkins P. F., Murray N., Quataert E., Thompson T. A., 2010, MNRAS, 401, L19

Hopkins P. F., Quataert E., Murray N., 2011, MNRAS, 417, 950

Hopkins P. F., Quataert E., Murray N., 2012a, MNRAS, 421, 3488

Hopkins P. F., Quataert E., Murray N., 2012b, MNRAS, 421, 3522

Hopkins P. F., Narayanan D., Murray N., 2013, MNRAS, 432, 2647

Hopkins P. F., Keres D., Onorbe J., Faucher-Giguere C.-A., Quataert E., Murray N., Bullock J. S., 2014, MNRAS, 445, 581

Hopkins P. F., Torrey P., Faucher-Giguère C.-A., Quataert E., Murray N., 2016, MNRAS, 458, 816

Hopkins P. F. et al., 2017, preprint (arXiv:1702.06148)

Johnson L. C. et al., 2016, ApJ, 827, 33

Kennicutt R. C., Jr, 1998a, ARA\&A, 36, 189

Kennicutt R. C., Jr, 1998b, ApJ, 498, 541

Keto E., Ho L. C., Lo K.-Y., 2005, ApJ, 635, 1062

Kim C.-G., Ostriker E. C., 2015, ApJ, 802, 99

Kritsuk A. G., Norman M. L., Wagner R., 2011, ApJ, 727, L20

Kroupa P., 2002, Science, 295, 82

Kruijssen J. M. D., 2012, MNRAS, 426, 3008

Kruijssen J. M. D., Maschberger T., Moeckel N., Clarke C. J., Bastian N., Bonnell I. A., 2012, MNRAS, 419, 841

Krumholz M. R., 2014, Phys. Rep., 539, 49

Krumholz M. R., Gnedin N. Y., 2011, ApJ, 729, 36

Krumholz M. R., McKee C. F., 2005, ApJ, 630, 250

Krumholz M. R., Tan J. C., 2007, ApJ, 654, 304

Krumholz M. R., Thompson T. A., 2012, ApJ, 760, 155

Krumholz M. R., Thompson T. A., 2013, MNRAS, 434, 2329

Krumholz M. R., Klein R. I., McKee C. F., 2011, ApJ, 740, 74

Krumholz M. R., Dekel A., McKee C. F., 2012, ApJ, 745, 69

Lada C. J., Lada E. A., 2003, ARA\&A, 41, 57

Lada C. J., Margulis M., Dearborn D., 1984, ApJ, 285, 141

Larson R. B., 1969, MNRAS, 145, 271

Larson R. B., 1981, MNRAS, 194, 809

Lee E. J., Chang P., Murray N., 2015, ApJ, 800, 49

Lee E. J., Miville-Deschênes M.-A., Murray N. W., 2016, ApJ, 833, 229

Martizzi D., Faucher-Giguère C.-A., Quataert E., 2015, MNRAS, 450, 504

Mathieu R. D., 1983, ApJ, 267, L97

Mayer L., Kazantzidis S., Escala A., Callegari S., 2010, Nature, 466, 1082

Mayer L., Fiacconi D., Bonoli S., Quinn T., Roškar R., Shen S., Wadsley J., 2015, ApJ, 810, 51

McCrady N., Graham J. R., 2007, ApJ, 663, 844

Mouri H., Taniguchi Y., 2002, ApJ, 566, L17

Murray N., 2011, ApJ, 729, 133

Murray N., Quataert E., Thompson T. A., 2010, ApJ, 709, 191

Myers A. T., Klein R. I., Krumholz M. R., McKee C. F., 2014, MNRAS, 439,3420 
Nordlund Å. K., Padoan P., 1999, in Franco J., Carraminana A., eds, Proc. 2nd Guillermo Haro Conf., Interstellar Turbulence. Cambridge Univ. Press, Cambridge, Cambridge, p. 218

Orr M. et al., 2017, MNRAS, preprint (arXiv:1701.01788)

Ostriker E. C., Shetty R., 2011, ApJ, 731, 41

Padoan P., Nordlund A., Jones B. J. T., 1997, MNRAS, 288, 145

Padoan P., Haugbølle T., Nordlund Å., 2012, ApJ, 759, L27

Parra R., Conway J. E., Diamond P. J., Thrall H., Lonsdale C. J., Lonsdale C. J., Smith H. E., 2007, ApJ, 659, 314

Penston M. V., 1969, MNRAS, 144, 425

Portegies Zwart S. F., McMillan S. L. W., 2002, ApJ, 576, 899

Portegies Zwart S. F., McMillan S. L. W., Gieles M., 2010, ARA\&A, 48, 431

Rafikov R. R., 2007, ApJ, 662, 642

Raskutti S., Ostriker E. C., Skinner M. A., 2016, ApJ, 829, 130

Rees M. J., 1976, MNRAS, 176, 483

Richings A. J., Schaye J., Oppenheimer B. D., 2014a, MNRAS, 440, 3349

Richings A. J., Schaye J., Oppenheimer B. D., 2014b, MNRAS, 442, 2780

Rodriguez C. L., Morscher M., Pattabiraman B., Chatterjee S., Haster C.-J., Rasio F. A., 2015, Phys. Rev. Lett., 115, 051101

Rodriguez C. L., Chatterjee S., Rasio F. A., 2016, Phys. Rev. D, 93, 084029

Rosdahl J., Teyssier R., 2015, MNRAS, 449, 4380

Ryon J. E. et al., 2015, MNRAS, 452, 525

Saitoh T. R., Daisaka H., Kokubo E., Makino J., Okamoto T., Tomisaka K., Wada K., Yoshida N., 2008, PASJ, 60, 667

Scoville N. et al., 2017, ApJ, 836, 66

Skinner M. A., Ostriker E. C., 2013, ApJS, 206, 21

Skinner M. A., Ostriker E. C., 2015, ApJ, 809, 187

Smith L. J., Gallagher J. S., 2001, MNRAS, 326, 1027

Smith R., Slater R., Fellhauer M., Goodwin S., Assmann P., 2011, MNRAS, 416, 383

Smith R., Goodwin S., Fellhauer M., Assmann P., 2013, MNRAS, 428, 1303

Solomon P. M., Rivolo A. R., Barrett J., Yahil A., 1987, ApJ, 319, 730

Springel V., 2005, MNRAS, 364, 1105

Springel V., 2010, MNRAS, 401, 791

Springel V., Hernquist L., 2003, MNRAS, 339, 289

Stone J. M., Gardiner T. A., Teuben P., Hawley J. F., Simon J. B., 2008, ApJS, 178, 137

Su K.-Y., Hopkins P. F., Hayward C. C., Faucher-Giguere C.-A., Keres D., Ma X., Robles V. H., 2017, MNRAS, 471, 144

Tan J. C., Krumholz M. R., McKee C. F., 2006, ApJ, 641, L121

Thompson T. A., Krumholz M. R., 2016, MNRAS, 455, 334

Thompson T. A., Quataert E., Murray N., 2005, ApJ, 630, 167

Torrey P., Hopkins P. F., Faucher-Giguère C.-A., Vogelsberger M., Quataert E., Kereš D., Murray N., 2017, MNRAS, 467, 2301

Troland T. H., Crutcher R. M., 2008, ApJ, 680, 457

Tsang B. T.-H., Milosavljević M., 2015, MNRAS, 453, 1108

Tsang B. T.-H., Milosavljevic M., 2017, preprint (arXiv:1709.07539)

Tutukov A. V., 1978, A\&A, 70, 57

Utreras J., Becerra F., Escala A., 2016, ApJ, 833, 13

Vazquez-Semadeni E., 1994, ApJ, 423, 681

Vink J. S., de Koter A., Lamers H. J. G. L. M., 2001, A\&A, 369, 574

Vutisalchavakul N., Evans N. J., II, Heyer M., 2016, ApJ, 831, 73

Wilson C. D., Harris W. E., Longden R., Scoville N. Z., 2006, ApJ, 641, 763

Wu J., Evans N. J., II, Gao Y., Solomon P. M., Shirley Y. L., Vanden Bout P. A., 2005, ApJ, 635, L173

Wu J., Evans N. J., II, Shirley Y. L., Knez C., 2010, ApJS, 188, 313

Yoast-Hull T. M., Gallagher J. S., Zweibel E. G., 2016, MNRAS, 457, L29

Zhang D., Davis S. W., 2017, ApJ, 839, 54

\section{APPENDIX A: CODE TESTS}

\section{A1 Convergence and consistency}

The methods for cooling, star formation, and feedback used in this paper have been tested in previous studies of galactic-scale simulations resolving spatial scales of $\sim 1 \mathrm{pc}$ and masses $>10^{3} \mathrm{M}_{\odot}$.

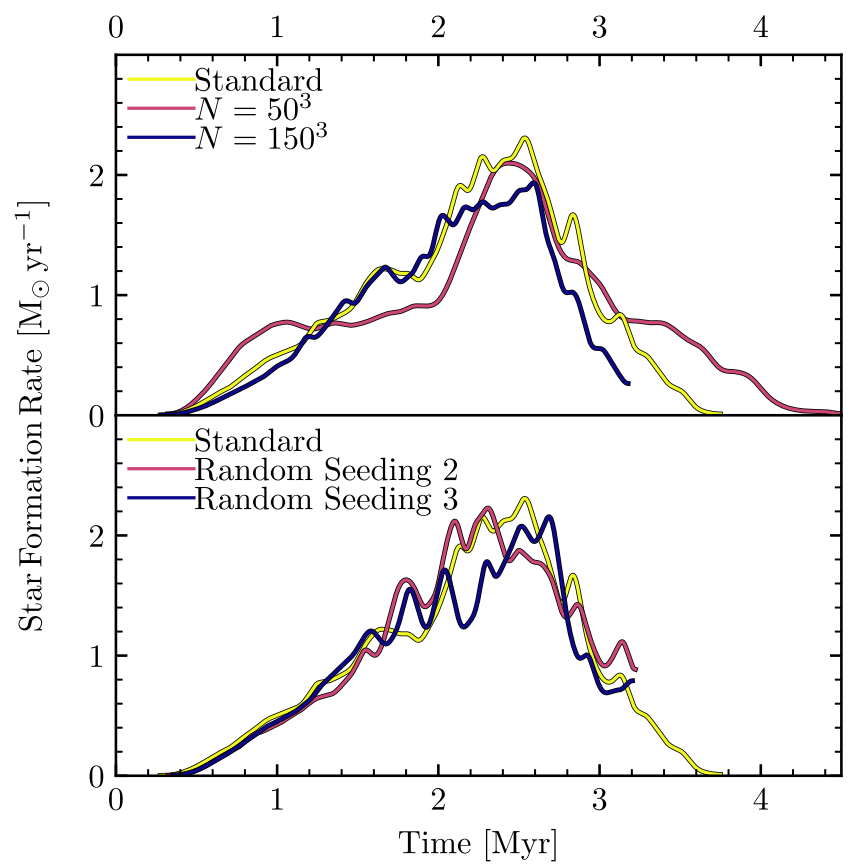

Figure A1. Star formation histories of test runs with parameters $M=$ $10^{7} \mathrm{M}_{\odot}$ and $R=50 \mathrm{pc}$. Top: Convergence tests with particle number varied from $50^{3}$ to $200^{3}$. Bottom: Consistency tests using three different random seeds for the initial perturbations.

However, their behaviour at the higher resolutions of these simulations has been much less well-studied. It is therefore necessary to determine how the simulation behaviour depends (1) upon mass and spatial resolution, (2) upon the particular random seeding in the initial conditions, and (3) upon the particular physics included and parameters chosen. Because the star formation histories (SFH) are the main data of interest, we shall focus on the effects of these choices on the SFH as a proxy for the behaviour of the simulation as a whole.

We choose the parameters $R=50 \mathrm{pc}, M=10^{7} \mathrm{M}_{\odot}$ as the point in parameter space at which to investigate these questions. Because all runs are qualitatively identical with only differences in numerical scalings, the conclusions drawn for these parameters should apply across our parameter space, obviating the need to perform the tests at all points. We vary the particle number from $50^{3}$ to $150^{3}$ to isolate resolution effects. Because we use adaptive softening, the effective gravitational force resolution naturally follows mass resolution with no need for manual tuning. To assess the effect of the random velocity seeding, we compare runs from three random realizations at the standard resolution and with standard physics.

From the first panel of Fig. A1 it is evident that mass resolution does have certain systematic effects upon the computed SFH: in particular, low-resolution runs have a SFR which is greater at early times. This is an artefact the cutoff in the turbulent length scale that can be followed before the turbulent Jeans mass is no longer resolved. A gas structure that is well-resolved and supported against its self-gravity by internal motions at high resolution may not be considered so if down-sampled to low resolution where it consists only of a few particles. Thus, in the absence of any feedback moderation, as is the case at early times, the SFR will rise sooner at low resolution. While this resolution effect is conspicuous, it apparently does not have a strong effect upon the integrated SFE.

The variation in SFE due to resolution is in fact comparable to the variation arising from different random seedings at fixed 
resolution, visible in panel 2 of Fig. 3. In both cases, the mass of gas converted to stars varies only by $\sim 1$ per cent between runs. We therefore conclude that the star formation efficiencies computed as the central result of this study are consistent between runs with the same physical parameters. As discussed in the main text, our results concerning SFE can be understood in terms of simple force balance considerations. As such, it is not surprising that the SFE should converge rapidly and be robust with respect to perturbations.

\section{A2 Radiation pressure}

In our survey of the effects of different physics (Section 4.1), it was found that radiation pressure was most responsible for the moderation of star formation. Therefore, it is particularly important to test the robustness of the radiative transfer prescription we have used. Radiation pressure is treated with a combination of shortranged, local coupling within the kernel encompassing a star particle's nearest neighbouring gas particles (most importantly handing single-scattered UV photons), and a long-ranged component treated in the optically thin approximation (mainly handling reprocessed IR photons). The estimated local extinction around a particle relies upon an estimate of the local column density $\Sigma_{\text {eff }}$ obtained by a Sobolev approximation; for details see Hopkins et al. (2017).

To test the sensitivity of our results to this local extinction approximation, we both increased and decreased the estimated $\Sigma_{\text {eff }}$ by a factor of 10 in our fiducial $10^{7} \mathrm{M}_{\odot}, 50 \mathrm{pc}$ run at $50^{3}$ resolution. The resulting star formation histories are shown in Fig. A2. Increasing $\Sigma_{\text {eff }}$ by a factor of 10 had very little effect on the star formation history. This is because the local extinction fraction $1-\exp \left(\Sigma_{\text {eff }} \kappa_{\mathrm{UV}}\right)$ is typically already quite close to 1 in the default run. Decreasing $\Sigma_{\text {eff }}$ by a factor of 10 reduced the peak SFR by roughly a factor of 2 , and decreased the final SFE from 0.32 to 0.23 , as leakage of UV photons from the local kernel is increased. We conclude that the SFE results of this paper do have some amount of sensitivity to the assumed geometric factor in the prescription for $\Sigma_{\text {eff }}$, but this sensitivity is quite sub-linear: variations of a factor of 10 lead to SFE variations within a factor of 2 .

Finally, we also performed a series of radiation pressure-only tests with a cloud of mass $5 \times 10^{4} \mathrm{M}_{\odot}$ and radius $15 \mathrm{pc}$, with a statistically isotropic solenoidal initial turbulent velocity field scaled to give an initial virial parameter of 2, emulating the setup in Raskutti et al. (2016). At mass resolutions at which the formation of dense

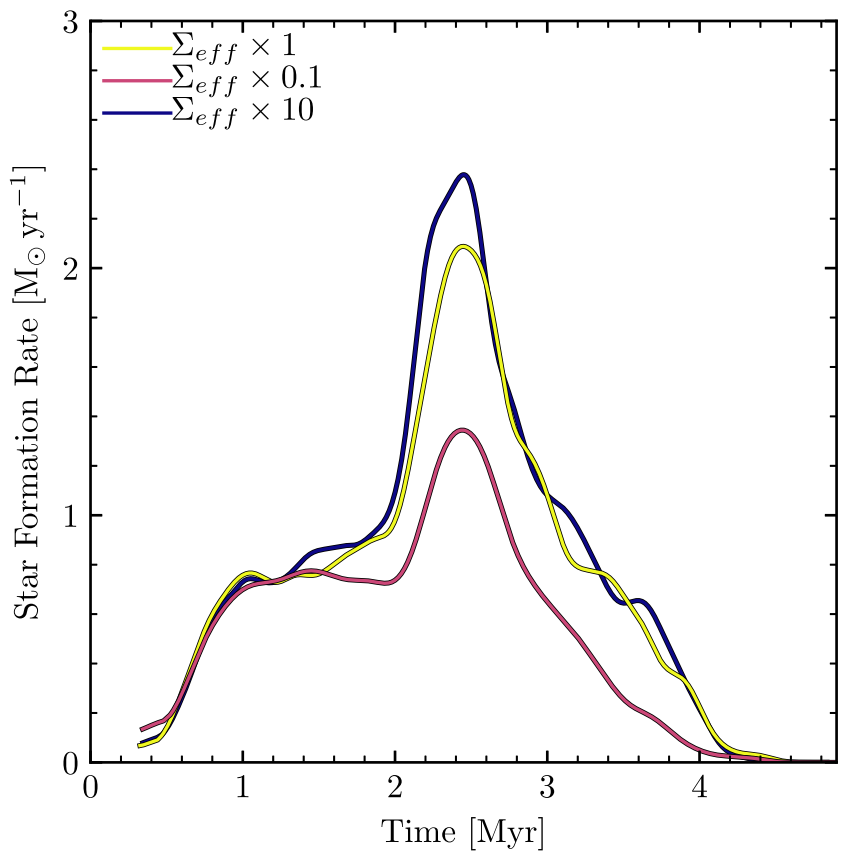

Figure A2. Effect of varying the local extinction column density estimator $\Sigma_{\text {eff }}$ by factors of 0.1 and 10 in our treatment of radiation pressure.

protostellar envelopes starts to be resolved $\left(\ll 1 \mathrm{M}_{\odot}\right)$, one might worry that some qualitative change in the nature of the density field would affect the local column density estimates in such a way that the net photon momentum budget at large is affected, and hence the SFE. We ran this test with particle numbers of $12^{3}, 25^{3}, 50^{3}$, and $100^{3}$, and obtained $\epsilon_{\text {int }}$ of $0.082,0.052,0.042$, and 0.040 , respectively, suggesting convergence. As with our convergence test with all physics enabled (A1), the SFE tends to converge from above; the star formation criterion is in some sense stricter at higher resolution, as local velocity gradients supporting against gravitational collapse are better-resolved.

This paper has been typeset from a $\mathrm{T}_{\mathrm{E}} \mathrm{X} / \mathrm{L} \mathrm{T} \mathrm{E} \mathrm{X}$ file prepared by the author. 